\title{
C-13 位烷氧基赤霉素衍生物的合成与抗肿瘤活性研究
}

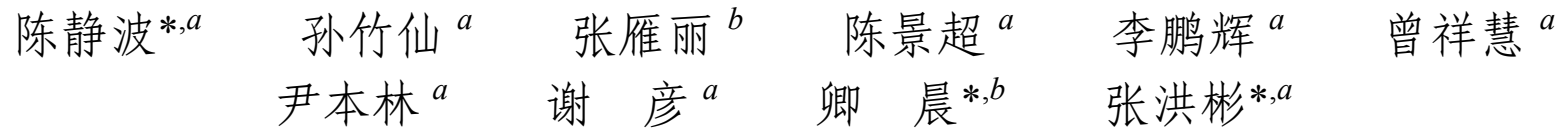 \\ ( ${ }^{a}$ 云南大学化学科学与工程学院 自然资源药物化学教育部重点实验室 昆明 650091) \\ $\left({ }^{b}\right.$ 昆明医科大学 云南省自然资源药理重点实验室 昆明 650031)
}

\begin{abstract}
摘要 设计并合成了系列 $\mathrm{C}-13$ 位为醚结构且在 $\mathrm{A}$ 环和 $\mathrm{D}$ 环有两个 $\alpha, \beta$-不饱和酮结构的赤霉素衍生物. 采用噻唑蓝 (MTT)法评价了其对人肺腺癌细胞(A549)、人肝癌细胞(HepG2)、人胃癌细胞(MKN28)以及结肠癌细胞(HT29)的体外生 长抑制作用, 结果表明: 此类化合物都有较强的体外抗肿瘤活性, 其中化合物 27 的活性最强(对人胃癌细胞 MKN28 的 $\mathrm{IC}_{50}=0.21 \mu \mathrm{mol} \cdot \mathrm{L}^{-1}$ ), 优于阳性对照药物顺铂(DDP).
\end{abstract}

关键词 赤霉素; 醚; $\alpha, \beta$-不饱和酮; 抗肿瘤

\section{Synthesis and Anticancer Activities of C-13 Etheral Gibberellin Derivatives}

\author{
Chen, Jingbo*,a \\ Sun, Zhuxian ${ }^{a}$ \\ Zhang, Yanli ${ }^{b}$ \\ Chen, Jingchao ${ }^{a}$ \\ Li, Penghui $^{a}$ \\ Zeng, Xianghui ${ }^{a}$ \\ Yin, Benlin ${ }^{a}$ \\ $\mathrm{Xie}, \mathrm{Yan}^{a}$ \\ Qing, Chen ${ }^{*, b}$ \\ Zhang, Hongbin ${ }^{*, a}$ \\ ( ${ }^{a}$ Key Laboratory of Medicinal Chemistry for Natural Resource, Ministry of Education, School of Chemical Science and \\ Technology, Yunnan University, Kunming 650091) \\ ( ${ }^{b}$ Yunnan Key Laboratory of Pharmacology for Natural Products, Kunming Medical University, Kunming 650031)
}

\begin{abstract}
C}$-13 ethereal gibberellin derivatives bearing two $\alpha, \beta$-unsaturated ketones in A and D rings have been designed and synthesized. In vitro studies demonstrated that a number of the $\mathrm{C}-13$ ethereal gibberellin derivatives showed strong anticancer activities in thiazolylblue (MTT) assay towards four human cancer cell lines including HT29, A-549, HepG2 and MKN28. Compound 27 was found to be the most potent compound $\left(\mathrm{IC}_{50}=0.21 \mu \mathrm{mol} \cdot \mathrm{L}^{-1}\right.$ against $\left.\mathrm{MKN} 28\right)$ and more active than the positive control (DDP).
\end{abstract}

Keywords gibberellin; ether; $\alpha, \beta$-unsaturated ketone; anticancer

赤霉素是一种广泛存在于高等植物中的二萜酸类 物质, 对植物的生长和发育有重要的调节作用. 赤霉酸 $\left(\mathrm{GA}_{3}\right)$ 是一种已经商业化发酵生产并在世界范围内大量 使用的农用植物生长调节剂 ${ }^{[1]}$. 从生源途径来看, 赤霉 酸类化合物是由存在于植物、微生物和海洋生物中的另 一大类天然产物对映贝壳杉烯代谢转化而来 ${ }^{[2]}$. 一些对 映贝壳杉烯类物质具有诸如抗菌、抗肿瘤、抗炎、拒食 等生物活性 ${ }^{[3]}$. 图 1 所示化合物为具有此类生物活性的
代表性二萜类物质. 据文献报道，具有 $\alpha, \beta$-不饱和酮结 构的对映贝壳杉烯类物质为一类迈克尔反应受体分子, 作为 NF- $\mathrm{kB}$ 核转录因子抑制剂 ${ }^{[4]}$ ，对多种肿瘤细胞显示 出良好的抑制活性 ${ }^{[5]}$. 我们在赤霉素衍生化与抗肿瘤活 性研究中发现, 整合到赤霉素中的 $\alpha, \beta$-不饱和酮结构是 抗肿瘤作用的关键药效团 ${ }^{[6]}$. 本文报道 $\mathrm{C}-13$ 位烷氧代的 含双 $\alpha, \beta$-不饱和酮结构赤䨝素衍生物的合成与抗肿瘤活 性研究.

\footnotetext{
*E-mail: zhanghb@ynu.edu.cn or zhanghbyd@gmail.com Received September 23, 2013; revised December 5, 2013; published online December 13, 2013.

Project supported by the National Natural Science Foundation of China (Nos. 21062029, 20925205), the Natural Science Foundation of Yunan Province (Nos. 2009CD007, 2010GA014) and the National Basic Research Program of China (973 Program, No. 2009CB522300).

国家自然科学基金(Nos. 21062029, 20925205)、云南省自然科学基金(Nos. 2009CD007, 2010GA014)及国家基础研究(973 项目, No. 2009CB522300)资助 项目.
} 


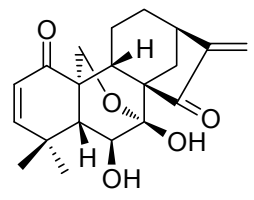

Eriocalycin B (1)

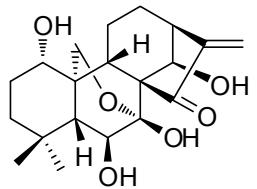

Oridonin (2)

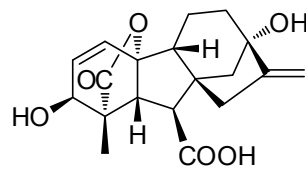

Gibberellin $\mathrm{GA}_{3}$ (3)

图 1 代表性四环二萜类化合物 $\mathbf{1} \sim 3$

Figure 1 Representative tetracyclic diterpenes $1 \sim 3$

\section{1 结果与讨论}

以易得的商品化原料赤霉素 $\mathrm{GA}_{3}(\mathbf{3})$ 为原料, 经酯 化反应转化为相应的甲酯 $\mathbf{4}$ 或苯甲酯 $\mathbf{5}^{[7]}$. 然后在二氧 化硒和过氧化叔丁醇的作用下进行 D 环烯丙位氧化引 入一个羟基得到三醇化合物 $\mathbf{6}$ 和 7 (Scheme 1$)^{[8]}$.

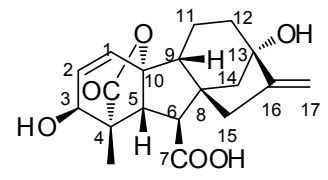

3
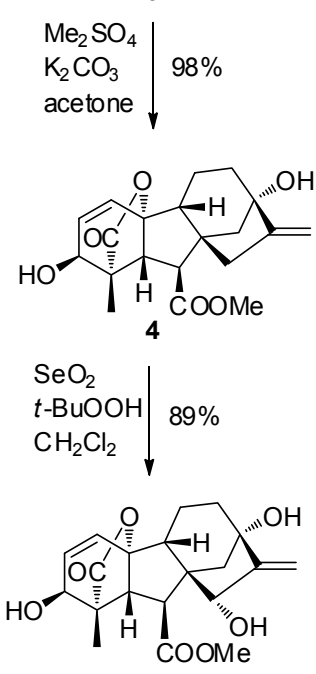

7

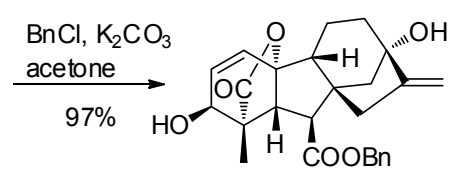

5
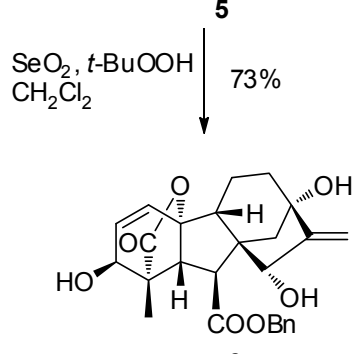

6

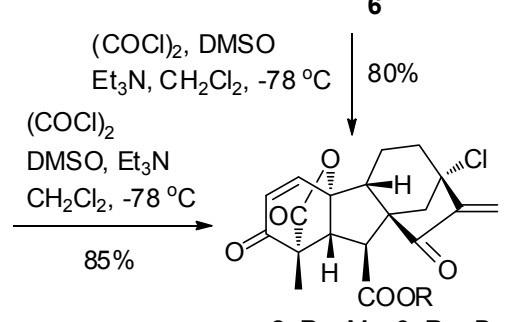

8, $\mathrm{R}=\mathrm{Me} ; \mathbf{9}, \mathrm{R}=\mathrm{Bn}$
Scheme 1

接下来我们尝试用 PDC(重铬酸吡啶盐)、活性二氧 化镇等作为氧化剂对化合物 $\mathbf{6}$ 和 7 的烯丙位醇进行氧化, 未能转化为期望的含 $\alpha, \beta$-不饱和酮结构的衍生物. 最后 经 Swern 氧化成功地将化合物 $\mathbf{6}$ 和 7 转化为含有双 $\alpha, \beta$ 不饱和酮结构的赤霉素衍生物 8 和 9 (Scheme 1) ${ }^{[9]}$. C-13 位的羟基在 Swern 氧化条件下同时发生了氯代反应. 产 物经核磁共振氢谱 $\left({ }^{1} \mathrm{H}\right.$ NMR $)$ 和碳谱 $\left({ }^{13} \mathrm{C}\right.$ NMR)、质谱
(LRMS 和 HRMS) 以及 $\mathrm{X}$ 射线晶体结构分析确证了结构 (图 2).

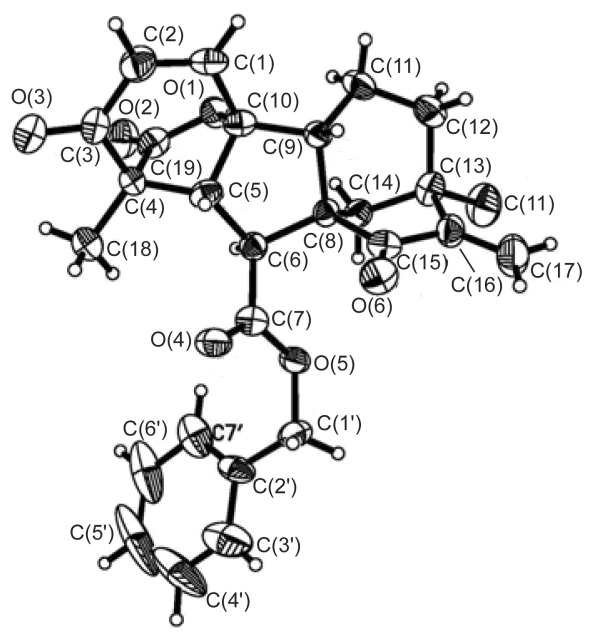

图 2 化合物 9 的 X 射线衍射晶体结构分析

Figure 2 X-ray crystallography of compound 9

在赤霉素衍生化及抗肿瘤活性的前期研究中 ${ }^{[6]}$, 发 现化合物 8 和 9 具有较强的肮肿瘤活性和较低的毒性, 但在有水条件下不稳定. 最近的研究结果表明 C-13 位 醚结构(烷氧基取代)的对映贝壳杉烯二萜类化合物对一 些肿瘤具有显著的抑制活性 ${ }^{[10]}$. 为增强该类化合物的 稳定性, 将化合物 8 和 9 的 C-13 位氯原子经取代反应转 化为羟基、甲氧基和乙氧基得到化合物 10 12, 19 21, 这些化合物在含水溶液中的稳定性大大提高，仍具有良 好的抗肿瘤活性. 为了更系统的研究 C-13 位烷氧基赤 霉素的构效关系, 又合成了 13 位为正(异)丙基、正(异) 丁基、正(异)戍基的衍生物(表 1).

表 1 C-13 烷氧代赤霉素衍生物的合成 ${ }^{a}$

Table 1 Synthesis of new gibberellin derivatives bearing C-13 ethereal moiety
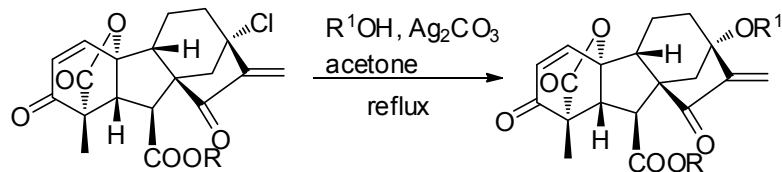

8, $\mathrm{R}=\mathrm{Me} ; \mathbf{9}, \mathrm{R}=\mathrm{Bn}$

$10 \sim 27$

\begin{tabular}{ccc||ccc}
\hline $\mathrm{GA}$ & $\mathrm{R}$ & $\mathrm{R}^{1}$ & $\mathrm{GA}$ & $\mathrm{R}$ & $\mathrm{R}^{1}$ \\
\hline $\mathbf{1 0}$ & $\mathrm{Me}$ & $\mathrm{H}$ & $\mathbf{1 9}$ & $\mathrm{Bn}$ & $\mathrm{H}$ \\
$\mathbf{1 1}$ & $\mathrm{Me}$ & $\mathrm{Me}$ & $\mathbf{2 0}$ & $\mathrm{Bn}$ & $\mathrm{Me}$ \\
$\mathbf{1 2}$ & $\mathrm{Me}$ & $\mathrm{Et}$ & $\mathbf{2 1}$ & $\mathrm{Bn}$ & $\mathrm{Et}$ \\
$\mathbf{1 3}$ & $\mathrm{Me}$ & $i-\mathrm{Pr}$ & $\mathbf{2 2}$ & $\mathrm{Bn}$ & $i-\mathrm{Pr}$ \\
$\mathbf{1 4}$ & $\mathrm{Me}$ & $n-\mathrm{Pr}$ & $\mathbf{2 3}$ & $\mathrm{Bn}$ & $n-\mathrm{Pr}$ \\
$\mathbf{1 5}$ & $\mathrm{Me}$ & $n-\mathrm{Bu}$ & $\mathbf{2 4}$ & $\mathrm{Bn}$ & $n-\mathrm{Bu}$ \\
$\mathbf{1 6}$ & $\mathrm{Me}$ & $i-\mathrm{Bu}$ & $\mathbf{2 5}$ & $\mathrm{Bn}$ & $i-\mathrm{Bu}$ \\
$\mathbf{1 7}$ & $\mathrm{Me}$ & $n-\mathrm{Pt}$ & $\mathbf{2 6}$ & $\mathrm{Bn}$ & $n-\mathrm{Pt}$ \\
$\mathbf{1 8}$ & $\mathrm{Me}$ & $i-\mathrm{Pt}$ & $\mathbf{2 7}$ & $\mathrm{Bn}$ & $i-\mathrm{Pt}$ \\
\hline${ }^{a} \mathrm{Pt}$
\end{tabular}


将 C-13 位氯代的赤霉素化合物 8 和 9 与碳酸银和 水或醇作用, 转化为相应的 C-13 位羟基或烷氧基类衍 生物. 经此方法共合成了 18 个目标化合物(表 1 ).

得到 C-13 位醚结构(烷氧基取代)的赤霉素衍生物 后, 我们进行了生物活性测试. 按照文献方法 ${ }^{[11]}$, 对所 有合成制备的赤霉素衍生物对人肺腺癌细胞(A549)、人 肝癌细胞(HepG2)、人胃癌细胞(MKN28)以及结肠癌细 胞(HT29)共四种人肿瘤细胞的细胞毒活性进行了评价, 结果见表 $2\left[\mathrm{IC}_{50}\right.$ 为半数生长抑制浓度, 顺铂(DDP)和含 有相同药效团(双 $\alpha, \beta$-不饱和酮)的毛蓦乙素(Eriocalycin $\mathrm{B}, 1)$ 作为活性对照化合物]. 在所有测试的赤霉素醚类 衍生物中, 化合物 13 对人结肠癌细胞(HT29)表现出最 强的抑制活性 $\left(\mathrm{IC}_{50}=0.24 \mu \mathrm{mol} \cdot \mathrm{L}^{-1}\right)$, 而化合物 27 对人 胃癌细胞(MKN28)表现出最强的抑制活性 $\left(\mathrm{IC}_{50}=0.21\right.$ $\mu \mathrm{mol} \cdot \mathrm{L}^{-1}$ ). 化合物 $13,16,22,25$ 对结肠癌细胞(HT29)具 有较高细胞毒, 但对人肺腺癌细胞(A549)毒性很低, 显 示了一定的肿瘤选择性. 总体而言, 人结肠癌细胞 (HT29)和人胃癌细胞(MKN28) 对具有 C-13 位醚结构(烷 氧基取代)的赤霉素衍生物更敏感(表 2). 具有侧链的 C-13 烷基(异丙基、异丁基、异戊基)醚类赤霉素衍生物 比直链烷基醚类衍生物对测试所用的肿瘤细胞显示更 强的抑制活性，提示位阻较大的 C-13 位醚结构可能具 有更强的抗肿瘤活性.

表 2 C-13 烷氧代赤霉素衍生物体外细胞毒活性 ${ }^{a}$

Table 2 In vitro cytotoxic activities of gibberellins bearing a C-13 ethereal moiety

\begin{tabular}{|c|c|c|c|c|}
\hline GAs & HT29 & A549 & HepG2 & MKN28 \\
\hline 10 & $24.50 \pm 0.52$ & $33.87 \pm 0$ & $13.85 \pm 0.65$ & $14.74 \pm 0.32$ \\
\hline 11 & $31.00 \pm$ & $27.50 \pm$ & 32 & $1.32 \pm 0.35$ \\
\hline 12 & $13.67 \pm 0.45$ & $7.27 \pm 0.42$ & $>40$ & $33.11 \pm 0.62$ \\
\hline 13 & $4 \pm 0.62$ & $>40$ & $25.37 \pm 1.40$ & $6.89 \pm 0.35$ \\
\hline 14 & $28.22 \pm 2.20$ & $>40$ & $10.81 \pm 0.24$ & $12.35 \pm 0.56$ \\
\hline 15 & $0.90 \pm 0.30$ & $32.61 \pm 1.10$ & $9.61 \pm 0.25$ & $0.98 \pm 0.62$ \\
\hline 16 & $0.63 \pm 0.56$ & $>40$ & $3.82 \pm 0.46$ & $0.67 \pm 0.78$ \\
\hline 17 & $0.58 \pm 0.45$ & $28.19 \pm 0$ & $4.12 \pm 0$ & $0.33 \pm 0.15$ \\
\hline 18 & $0.26 \pm 0.60$ & $25.72 \pm 0$ & $6.64 \pm 0.50$ & $0.56 \pm 1.10$ \\
\hline 19 & $37.36 \pm 0.35$ & $10.70 \pm 2$ & $9.17 \pm 0.75$ & $11.00 \pm 1.25$ \\
\hline 20 & $22.37 \pm 0.18$ & $13.02 \pm 0$ & $16.19 \pm 0.79$ & $0.35 \pm 0.23$ \\
\hline 21 & $11.09 \pm 0.45$ & $11.52 \pm 0.35$ & $14.10 \pm 0.54$ & $7.86 \pm 0.34$ \\
\hline 22 & $0.57 \pm 0.75$ & $>40$ & $8.80 \pm 0$ & $0.42 \pm 0.63$ \\
\hline 23 & $1.89 \pm 0.37$ & $>40$ & $7.59 \pm 0.64$ & $2.79 \pm 0.44$ \\
\hline 24 & $0.65 \pm 0.33$ & $28.34 \pm 1.45$ & $6.68 \pm 0.43$ & $3.01 \pm 0.25$ \\
\hline 25 & $1.24 \pm 0.38$ & $>40$ & $2.38 \pm 0.56$ & $0.70 \pm 0.45$ \\
\hline 26 & $0.60 \pm 0.45$ & $28.27 \pm 0.78$ & $2.58 \pm 0.45$ & $0.53 \pm 0.27$ \\
\hline 27 & $0.54 \pm 0.37$ & $24.96 \pm 0.55$ & $2.17 \pm 0.42$ & $0.21 \pm 0.35$ \\
\hline 8 & $1.14 \pm 0.52$ & $23.57 \pm 0.47$ & $15.57 \pm 0.33$ & $8.54 \pm 0.43$ \\
\hline 9 & $2.10 \pm 0.35$ & $13.45 \pm 0.57$ & $22.05 \pm 0.37$ & $10.67 \pm 0.45$ \\
\hline DDP & $3.42 \pm 0.55$ & $10.29 \pm 0.43$ & $3.10 \pm 0.64$ & $1.25 \pm 0.32$ \\
\hline 1 & $0.26 \pm 0.14$ & $0.40 \pm 0.31$ & $0.53 \pm 0.22$ & $0.14 \pm 0.24$ \\
\hline
\end{tabular}

\section{2 结论}

合成了 18 个含双 $\alpha, \beta$-不饱和酮结构的 13-烷氧代赤 霉素衍生物, 并用改良 MTT 法对其体外抗肿瘤活性进 行了评估. 初步的构效关系: 此类赤霉素衍生物中, $\alpha, \beta$ 不饱和酩结构和 C-13 位醚结构都对抗肿瘤作用起关键 作用, C-13 位醚结构中不同的烷基不仅导致抗肿瘤活性 的差异，而且显示出一定的肿瘤类型选择性，具有侧链 的烷基(异丙基、异丁基、异戊基)醚类赤霉素衍生物比 直链烷基醚类衍生物对测试所用的肿瘤细胞显示更强 的抑制活性，提示位阻较大的 C-13 位醚结构可能具有 肿瘤类型选择性和更强的抗肿瘤活, 为深入研究此类化 合物的构效关系提供了较好的参考. 对抗肿瘤活性非常 好的化合物 27 的进一步生物活性和作用机制研究在进 行中.

\section{3 实验部分}

\section{1 仪器与试剂}

核磁共振氢谱 $\left({ }^{1} \mathrm{H}\right.$ NMR $)$ 和碳谱 $\left({ }^{13} \mathrm{C}\right.$ NMR $)$ 用 Bruker Avance 300 核磁共振仪测定，以四甲基硅烷(TMS)为内 标; 低分辩质谱(EI-MS) 在 VG Auto Spec- 3000 质谱仪 上测定，高分辨质谱(HRMS)在 AB QSTAR Pulsar 质谱 仪上测定; 熔点由国产北京泰克仪器设备有限公司生产 的 XT-4 型双目显微熔点测定仪测定，温度计未校正. 赤霉素 $\mathrm{GA}_{3}$ (Gibberellin $\mathrm{A}_{3}$; ent-3 $\alpha, 10 \beta$-13-trihydroxy-20norgibberella-1,16-diene-7,19-dioic acid 19,10-lactone)为 江苏丰源生物化工有限公司产品, 无水四氢呋喃经与金 属钠回流(二苯甲酮为指示剂)至深蓝色后重蒸获得, 无 水二氯甲烷经与氢化钻回流重蒸获得. 所用其它原料和 试剂购自 Acros, Aldrich, Fluka, 除特别指出之外, 均未 经纯化直接使用.

\section{2 实验方法}

\subsection{1 赤霉素衍生物的合成}

对映-3 $\alpha, 10 \beta, 13 \beta$-三羟基-20-失碳赤霉-1,16-二烯7,19-二羧酸 7-甲酯 19,10-内酯(4)：赤霉素 $\left(\mathrm{GA}_{3}, 1.0 \mathrm{~g}\right.$, $2.9 \mathrm{mmol}$ )溶于丙酮 $(20 \mathrm{~mL})$ 中, 然后在冰水浴冷却下加 入硫酸二甲酯 $(0.73 \mathrm{~g}, 5.8 \mathrm{mmol}, 2.0$ equiv. $)$ 和碳酸钾 ( $0.80 \mathrm{~g}, 5.8 \mathrm{mmol})$. 常温摚拌反应过夜后减压蒸出溶剂, 所得残余物中加入冰 $(20 \mathrm{~g})$ 和稀盐酸 $\left(2 \mathrm{~mol} \cdot \mathrm{L}^{-1}, 20 \mathrm{~mL}\right)$, 搅拌, 所得混合物用乙酸乙酯 $(20 \mathrm{~mL} \times 2)$ 萃取. 合并有 机相, 水洗 $(15 \mathrm{~mL} \times 2)$ 后, 再用饱和盐水 $(15 \mathrm{~mL})$ 洗. 经 无水硫酸钠干燥后, 过滤, 滤液经减压蒸干得粗品 $(1.02 \mathrm{~g})$. 未经纯化直接用于下一步反应. ${ }^{1} \mathrm{H}$ NMR (300 $\left.\mathrm{MHz}, \mathrm{CD}_{3} \mathrm{COCD}_{3}\right) \delta: 6.37(\mathrm{~d}, J=9.3 \mathrm{~Hz}, 1 \mathrm{H}), 5.89(\mathrm{dd}$, $J=3.5,9.3 \mathrm{~Hz}, 1 \mathrm{H}), 5.21(\mathrm{~s}, 1 \mathrm{H}), 4.88(\mathrm{~s}, 1 \mathrm{H}), 4.03(\mathrm{~d}, J=$ 
$3.5 \mathrm{~Hz}, 1 \mathrm{H}), 3.72$ (s, 3H), 3.25 (d, $J=10.7 \mathrm{~Hz}, 1 \mathrm{H}), 2.70$ (d, $J=10.7 \mathrm{~Hz}, 1 \mathrm{H}), 2.28 \sim 1.62(\mathrm{~m}, 10 \mathrm{H}), 1.50 \sim 1.38(\mathrm{~m}$, $1 \mathrm{H}), 1.16(\mathrm{~s}, 3 \mathrm{H}) ;{ }^{13} \mathrm{C}$ NMR $\left(75 \mathrm{MHz}, \mathrm{CD}_{3} \mathrm{COCD}_{3}\right) \delta$ : 178.9, 173.0, 158.5, 133.9, 132.7, 106.9, 91.1, 78.1, 70.1, 54.1, 53.4, 52.1, 51.7, 51.0, 45.5, 43.8, 39.7, 17.6, 14.9.

对映-3 $\alpha, 10 \beta, 13 \beta$-三羟基-20-失碳赤雱-1,16-二烯7,19-二羧酸 7-苯甲酯 19,10-内酯(5): 赤霉素 $\left(\mathrm{GA}_{3}, 1.0 \mathrm{~g}\right.$, $2.9 \mathrm{mmol}$ )溶于丙酮 $(20 \mathrm{~mL})$ 中, 然后在冰水浴冷却下加 入苯甲基溴 $(0.50 \mathrm{~g}, 2.9 \mathrm{mmol}, 1.0$ equiv.) 和碳酸钾 $(0.80$ g, $5.8 \mathrm{mmol})$. 常温傥拌反应过夜后减压蒸出溶剂, 所得 残余物中加入冰 $(20 \mathrm{~g})$ 和稀盐酸 $\left(2 \mathrm{~mol} \cdot \mathrm{L}^{-1}, 20 \mathrm{~mL}\right)$, 摚 拌, 所得混合物用乙酸乙酯 $(20 \mathrm{~mL} \times 4)$ 萃取. 合并有机 相, 水洗 $(15 \mathrm{~mL} \times 2)$ 后, 再用饱和盐水 $(15 \mathrm{~mL})$ 洗. 经无 水硫酸钠干燥后, 过滤, 滤液经减压蒸干得粗品(1.02 g). 未经纯化直接用于下一步反应. ${ }^{1} \mathrm{H}$ NMR $(300 \mathrm{MHz}$, $\left.\mathrm{CDCl}_{3}\right) \delta: 7.43 \sim 7.30$ (br s, $5 \mathrm{H}$ ), 6.31 (d, $J=9.0 \mathrm{~Hz}, 1 \mathrm{H}$ ), 5.89 (dd, $J=3.6,9.3 \mathrm{~Hz}, 1 \mathrm{H}), 5.23(\mathrm{~s}, 1 \mathrm{H}), 5.21$ (d, $J=$ $12.3 \mathrm{~Hz}, 1 \mathrm{H}), 5.13$ (d, $J=12.3 \mathrm{~Hz}, 1 \mathrm{H}), 4.89$ (s, 1H), $4.18 \sim 4.09(\mathrm{~m}, 1 \mathrm{H}), 3.22(\mathrm{~d}, J=10.8 \mathrm{~Hz}, 1 \mathrm{H}), 2.81(\mathrm{~d}, J=$ $10.8 \mathrm{~Hz}, 1 \mathrm{H}), 2.15 \sim 2.00(\mathrm{~m}, 6 \mathrm{H}), 1.98 \sim 1.60(\mathrm{~m}, 5 \mathrm{H})$, 1.19 (s, 3H); ${ }^{13} \mathrm{C}$ NMR $\left(75 \mathrm{MHz}, \mathrm{CDCl}_{3}\right) \delta: 178.2,171.8$, 156.9, 132.9, 132.3, 128.6, 128.4, 107.3, 90.3, 78.3, 69.8, $66.9,53.4,52.9,51.2,50.7,50.5,44.9,42.9,38.1,16.9$, 14.3.

对映- $3 \alpha, 10 \beta, 13 \beta, 15 \beta$-四羟基-20-失碳赤霉-1,16-二 烯-7,19-二羧酸 7-苯甲酯 19,10-内酯(6): 赤霉酸苯甲酯 $(\mathbf{5}, 436 \mathrm{mg}, 1.0 \mathrm{mmol})$ 溶于二氯甲烷 $(8 \mathrm{~mL})$ 中, 然后加入 二氧化硒(133.2 mg, $1.2 \mathrm{mmol}, 1.2$ equiv.)和过氧化叔丁 醇(70\%水溶液, $0.93 \mathrm{~g} / \mathrm{mL}, 0.32 \mathrm{~mL}, 2.3$ equiv.). 常温摚 拌 $16 \mathrm{~h}$ 后, 短硅胶柱过滤, 并用乙酸乙酯 $(80 \mathrm{~mL})$ 冲洗. 经无水硫酸钠干燥后, 过滤, 滤液经减压蒸干所得粗品 经硅胶柱层析纯化 $(200 \sim 300$ 目, 二氯甲烷/乙酸乙酯, $V: V=2: 1 \sim 1: 1$ ), 得浅黄色粘稠液体(328 $\mathrm{mg}, 73 \%$ ). ${ }^{1} \mathrm{H}$ NMR $\left(300 \mathrm{MHz}, \mathrm{CD}_{3} \mathrm{COCD}_{3}\right) \delta: 7.52 \sim 7.23(\mathrm{~m}, 5 \mathrm{H})$, $6.38(\mathrm{~d}, J=9.3 \mathrm{~Hz}, 1 \mathrm{H}), 5.86(\mathrm{dd}, J=3.6,9.3 \mathrm{~Hz}, 1 \mathrm{H})$, $5.32(\mathrm{~s}, 1 \mathrm{H}), 5.28(\mathrm{~s}, 1 \mathrm{H}), 5.14(\mathrm{~d}, J=12.6 \mathrm{~Hz}, 1 \mathrm{H}), 4.99$ (d, $J=12.6 \mathrm{~Hz}, 1 \mathrm{H}), 4.68(\mathrm{~d}, J=6.8 \mathrm{~Hz}, 1 \mathrm{H}), 4.17$ (d, $J=$ $4.6 \mathrm{~Hz}, 1 \mathrm{H}), 4.12 \sim 3.98(\mathrm{~m}, 2 \mathrm{H}), 3.82(\mathrm{~s}, 1 \mathrm{H}), 3.24(\mathrm{~d}, J=$ $10.1 \mathrm{~Hz}, 1 \mathrm{H}), 2.63(\mathrm{~d}, J=10.1 \mathrm{~Hz}, 1 \mathrm{H}), 2.37(\mathrm{~d}, J=10.8$ $\mathrm{Hz}, 1 \mathrm{H}), 2.00 \sim 1.62(\mathrm{~m}, 6 \mathrm{H}), 1.16(\mathrm{~s}, 3 \mathrm{H}) ;{ }^{13} \mathrm{C}$ NMR $(75$ $\left.\mathrm{MHz}, \mathrm{CD}_{3} \mathrm{COCD}_{3}\right) \delta: 178.9,173.6,162.6,137.5,133.9$, $132.8,129.1,128.7,128.5,111.6,91.3,78.2,76.9,70.3$, 66.9, 57.8, 54.5, 54.1, 49.6, 48.2, 42.8, 39.8, 18.0, 14.8; EIMS $m / z(\%): 452\left(\mathrm{M}^{+}, 0.25\right), 434(0.5), 406(0.4), 390$ (1.5), 372 (0.4), 344 (100), 343 (34), 325 (6), 298 (5), 281
(7), 271 (12), 253 (21), 237 (17), 225 (27), 209 (28), 201 (32), 181 (18), 149 (26), 135 (17), 121 (12), 108 (65); HRMS calcd for $\mathrm{C}_{26} \mathrm{H}_{28} \mathrm{O}_{7} \mathrm{Na}(\mathrm{M}+\mathrm{Na})^{+} 475.1732$; found 475.1728 .

对映- $3 \alpha, 10 \beta, 13 \beta, 15 \beta$-四羟基-20-失碳赤霉-1,16-二 烯-7,19-二羧酸 7-甲酯 19,10-内酯(7): 赤霉酸甲酯 (4, $360 \mathrm{mg}, 1.0 \mathrm{mmol})$ 溶于二氯甲烷 $(8 \mathrm{~mL})$ 中, 然后加入二 氧化硒(133.2 mg, $1.2 \mathrm{mmol}, 1.2$ equiv.)和过氧化叔丁醇 (70\%水溶液, $0.93 \mathrm{~g} / \mathrm{mL}, 0.32 \mathrm{~mL}, 2.3$ equiv.). 常温傥拌 $16 \mathrm{~h}$ 后, 短硅胶柱过滤, 并用乙酸乙酯 $(80 \mathrm{~mL})$ 冲洗. 经 无水硫酸钠干燥后, 过滤, 滤液经减压蒸干所得粗品经 硅胶柱层析纯化 $(200 \sim 300$ 目，二氯甲烷/乙酸乙酯, $V$ : $V=2: 1 \sim 1: 1$ ), 得浅黄色粘稠液体(335 mg, 89\%). ${ }^{1} \mathrm{H}$ NMR (300 MHz, CD $\left.\mathrm{CD}_{3} \mathrm{OD}\right) \delta: 6.41(\mathrm{~d}, J=9.3 \mathrm{~Hz}, 1 \mathrm{H}), 5.89$ (dd, $J=3.3,9.3 \mathrm{~Hz}, 1 \mathrm{H}), 5.39$ (s, 1H), 5.37 (s, $1 \mathrm{H}), 4.12$ (s, 1H), 4.03 (d, $J=3.3 \mathrm{~Hz}, 1 \mathrm{H}), 3.70$ (s, 3H), 3.21 (d, $J=9.9$ $\mathrm{Hz}, 1 \mathrm{H}), 2.62(\mathrm{~d}, J=9.9 \mathrm{~Hz}, 1 \mathrm{H}), 2.28(\mathrm{~d}, J=11.1 \mathrm{~Hz}$, 1H), $2.01 \sim 1.70(\mathrm{~m}, 6 \mathrm{H}), 1.22(\mathrm{~s}, 3 \mathrm{H}) ;{ }^{13} \mathrm{C}$ NMR $(75 \mathrm{MHz}$, $\left.\mathrm{CD}_{3} \mathrm{OD}\right) \delta: 180.8,175.8,161.9,134.1,133.2,112.5,92.7$, 78.6, 77.4, 70.7, 58.3, 55.1, 54.8, 52.5, 50.1, 48.7, 43.0, 39.8, 18.4, 14.8; EIMS $m / z$ (\%): $376\left(\mathrm{M}^{+}, 0.25\right), 358$ (3), 344 (49), 346 (11), 315 (5), 298 (7), 280 (6), 271 (10), 255 (16), 253 (26), 239 (11), 237 (24), 225 (28), 209 (27), 201 (21), 197 (19), 171 (15), 165 (15), 149 (100), 129 (12), 121 (12), 115 (10); HRMS calcd for $\mathrm{C}_{20} \mathrm{H}_{24} \mathrm{O}_{7} \mathrm{Na}(\mathrm{M}+\mathrm{Na})^{+}$ 399.1419; found 399.1427 .

对映-13 $\beta$-氯-3,15-二氧-10 $\beta$-羟基-20-失碳赤霉1,16-二烯-7,19-二羧酸 7-甲酯 19,10-内酯 (8): 草酰氯 $(0.57 \mathrm{~mL}, 1.5 \mathrm{~g} / \mathrm{mL}, 6.73 \mathrm{mmol}, 5.0$ equiv.)溶于二氯甲烷 $(5 \mathrm{~mL})$ 并冷却到 $-78{ }^{\circ} \mathrm{C}$. 搅拌下缓慢滴加二甲亚砜 ( $0.96 \mathrm{~mL}, 1.1 \mathrm{~g} / \mathrm{mL}, 13.52 \mathrm{mmol}, 10.0$ equiv.) 的二氯甲烷 $(5 \mathrm{~mL})$ 溶液. 在 $-78{ }^{\circ} \mathrm{C}$ 继续摚拌 $10 \mathrm{~min}$ 后, 滴加 7 (507 $\mathrm{mg}, 1.35 \mathrm{mmol})$ 的二氯甲烷 $(4 \mathrm{~mL})$ 和二甲亚砜 $(0.58 \mathrm{~mL})$ 溶液. 反应液继续摚拌 $15 \mathrm{~min}$ 后, 滴加三乙胺 $(4.06 \mathrm{~mL}$, $29.2 \mathrm{mmol}, 20.0$ equiv.), 随后撤去冷浴, 使反应液逐渐 升至室温. 加入冰 $(15 \mathrm{~g})$ 和水 $(25 \mathrm{~mL})$, 搅拌, 所得混合 物用乙酸乙酯 $(10 \mathrm{~mL} \times 2)$ 萃取. 合并有机相, $2 \mathrm{~mol} \cdot \mathrm{L}^{-1}$ 稀盐酸洗 $(10 \mathrm{~mL} \times 2)$ 后, 再用水 $(10 \mathrm{~mL} \times 2)$ 洗. 经无水 硫酸钠干燥后, 过滤, 滤液经减压蒸干所得粗品经硅胶 柱层析纯化(200 300 目, 正己烷/乙酸乙酯, $V: V=5$ ： $1 \sim 2: 1$ ), 得无色晶体(335 mg, 89\%). m.p. 139 140 ${ }^{\circ} \mathrm{C} ;{ }^{1} \mathrm{H}$ NMR (300 MHz, $\left.\mathrm{CDCl}_{3}\right) \delta: 7.18(\mathrm{~d}, J=9.4 \mathrm{~Hz}$, $1 \mathrm{H}), 6.23(\mathrm{~s}, 1 \mathrm{H}), 6.07(\mathrm{~d}, J=9.4 \mathrm{~Hz}, 1 \mathrm{H}), 5.87(\mathrm{~s}, 1 \mathrm{H})$, $3.63(\mathrm{~s}, 3 \mathrm{H}), 3.62(\mathrm{~d}, J=10.2 \mathrm{~Hz}, 1 \mathrm{H}), 2.82(\mathrm{~d}, J=10.2$ $\mathrm{Hz}, 1 \mathrm{H}), 2.79$ (d, $J=11.4 \mathrm{~Hz}, 1 \mathrm{H}), 2.65$ (dd, $J=8.1,13.6$ 
$\mathrm{Hz}, 1 \mathrm{H}), 2.50(\mathrm{~d}, J=11.4 \mathrm{~Hz}, 1 \mathrm{H}), 2.40 \sim 2.08(\mathrm{~m}, 3 \mathrm{H})$, $1.92 \sim 1.82(\mathrm{~m}, 1 \mathrm{H}), 1.33(\mathrm{~s}, 3 \mathrm{H}) ;{ }^{13} \mathrm{C} \mathrm{NMR}(75 \mathrm{MHz}$, $\left.\mathrm{CDCl}_{3}\right) \delta: 200.3,191.0,172.6,170.2,150.7,145.8,129.7$, 121.3, 89.1, 65.9, 65.4, 61.2, 60.5, 52.4, 48.8, 47.2, 41.5, 39.8, 18.0, 11.9; EIMS m/z (\%): $391\left(\mathrm{M}^{+}+1,4\right), 390\left(\mathrm{M}^{+}\right.$, 4), 361 (16), 360 (43), 358 (100), 326 (31), 303 (16), 286 (42), 267 (43), 251 (50), 236 (52), 222 (40), 217 (23), 213 (15), 195 (13), 165 (29), 152 (20), 141 (22), 128 (40), 115 (50); HRMS calcd. for $\mathrm{C}_{20} \mathrm{H}_{19} \mathrm{ClO}_{6} \mathrm{Na}(\mathrm{M}+\mathrm{Na})$ 413.0767, found 413.0781.

对映-13 $\beta$-氯-3,15-二氧- $10 \beta$ - 羟基-20-失碳赤霉1,16-二烯-7,19-二羧酸 7-苯甲酯 19,10-内酯(9): 草酰 氯( $(0.57 \mathrm{~mL}, 1.5 \mathrm{~g} / \mathrm{mL}, 6.73 \mathrm{mmol}, 5.0$ equiv.)溶于二氯甲 烷 $(5 \mathrm{~mL})$ 并冷却到 $-78{ }^{\circ} \mathrm{C}$. 搅拌下缓慢滴加二甲亚砜 (0.96 mL, $1.1 \mathrm{~g} / \mathrm{mL}, 13.52 \mathrm{mmol}, 10.0$ equiv.)的二氯甲烷 $(5 \mathrm{~mL})$ 溶液. 在 $-78{ }^{\circ} \mathrm{C}$ 继续摚拌 $10 \mathrm{~min}$ 后, 滴加 6 (611 $\mathrm{mg}, 1.35 \mathrm{mmol})$ 的二氯甲烷 $(4 \mathrm{~mL})$ 和二甲亚砜 $(0.58 \mathrm{~mL})$ 溶液. 反应液继续搅拌 $15 \mathrm{~min}$ 后, 滴加三乙胺 $(4.06 \mathrm{~mL}$, $29.2 \mathrm{mmol}, 20.0$ equiv.), 随后撤去冷浴, 使反应液逐渐 升至室温. 加入冰 $(15 \mathrm{~g})$ 和水 $(25 \mathrm{~mL})$, 搅拌, 所得混合 物用乙酸乙酯 $(10 \mathrm{~mL} \times 2)$ 萃取. 合并有机相, $2 \mathrm{~mol} \cdot \mathrm{L}^{-1}$ 稀盐酸洗 $(10 \mathrm{~mL} \times 2)$ 后, 再用水 $(10 \mathrm{~mL} \times 2)$ 洗. 经无水 硫酸钠干燥后, 过滤, 滤液经减压蒸干所得粗品经硅胶 柱层析纯化 $(200 \sim 300$ 目, 正己烷/乙酸乙酯, $V: V=5$ : $1 \sim 2 ： 1$ ), 得无色针状固体(508 mg, 80\%). m.p. 177 $178{ }^{\circ} \mathrm{C} ;{ }^{1} \mathrm{H}$ NMR $\left(300 \mathrm{MHz}, \mathrm{CDCl}_{3}\right) \delta: 7.45 \sim 7.28(\mathrm{~m}$, $5 \mathrm{H}), 7.28(\mathrm{~d}, J=9.6 \mathrm{~Hz}, 1 \mathrm{H}), 6.24(\mathrm{~s}, 1 \mathrm{H}), 6.15$ (d, $J=9.6$ $\mathrm{Hz}, 1 \mathrm{H}), 5.93$ (s, 1H), 5.14 (s, 2H), 3.72 (d, $J=10.2 \mathrm{~Hz}$, $1 \mathrm{H}), 2.95$ (d, $J=10.2 \mathrm{~Hz}, 1 \mathrm{H}), 2.86$ (d, $J=11.4 \mathrm{~Hz}, 1 \mathrm{H})$, $2.72(\mathrm{dd}, J=7.2,13.2 \mathrm{~Hz}, 1 \mathrm{H}), 2.54$ (d, $J=11.4 \mathrm{~Hz}, 1 \mathrm{H})$, $2.48 \sim 2.12(\mathrm{~m}, 3 \mathrm{H}), 2.02 \sim 1.92(\mathrm{~m}, 1 \mathrm{H}), 1.41(\mathrm{~s}, 3 \mathrm{H}) ;{ }^{13} \mathrm{C}$ NMR (75 MHz, $\left.\mathrm{CDCl}_{3}\right) \delta: 200.3,191.0,172.6,169.5$, $150.8,145.7,134.9,129.7,128.6,128.6,121.1,89.1,67.5$, $65.9,65.4,61.2,60.6,49.0,47.3,41.6,39.7,18.0,11.9$; EIMS $m / z$ (\%): 363 (3), 362 (13), 360 (24), 334 (4), 333 (16), 331 (45), 286 (8), 280 (17), 267 (45), 249 (30), 223 (34), 221 (18), 208 (12), 195 (10), 181 (5), 165 (10), 158 (7), 128 (12), 115 (15), 107 (36), 91 (100); HRMS calcd for $\mathrm{C}_{26} \mathrm{H}_{23} \mathrm{ClO}_{6} \mathrm{Na}(\mathrm{M}+\mathrm{Na})^{+}$489.1080, found 489.1076.

对映-10 $\beta, 13 \beta$-二羊基-3,15-二氧-20-失碳赤霉-1,16二烯-7,19-二羧酸 7-甲酯 19,10-内酯(10): 赤䨘素衍生 物 8 (391 mg, $1.0 \mathrm{mmol})$ 溶于丙䣶 $(8 \mathrm{~mL})$ 和水 $(2 \mathrm{~mL})$ 中, 加入碳酸银(276 mg, $1.0 \mathrm{mmol}, 1.0$ equiv.), 搅拌回流 24 h. 减压蒸去溶剂, 残留物加入水 $(50 \mathrm{~mL})$, 用乙酸乙酯 $(15 \mathrm{~mL} \times 3)$ 萃取, 经无水硫酸钠干燥后, 过滤, 滤液经
减压蒸干所得粗品经硅胶柱层析纯化(200 300 目，正 己烷/乙酸乙酯, $V: V=5: 1 \sim 2: 1)$, 得白色固体(253 mg, 67.9\%). m.p. 231 232 ${ }^{\circ} \mathrm{C}$; ${ }^{1} \mathrm{H}$ NMR (300 MHz, $\left.\mathrm{CDCl}_{3}\right) \delta: 7.20(\mathrm{~d}, J=9.3 \mathrm{~Hz}, 1 \mathrm{H}), 6.13$ (s, 1H), 6.08 (d, $J=9.3 \mathrm{~Hz}, 1 \mathrm{H}), 5.70$ (s, 1H), 3.67 (d, $J=10.2 \mathrm{~Hz}, 1 \mathrm{H})$, 3.64 (s, 3H), 2.83 (d, $J=10.2 \mathrm{~Hz}, 1 \mathrm{H}), 2.54$ (d, $J=10.8$ $\mathrm{Hz}, 1 \mathrm{H}), 2.36 \sim 2.18(\mathrm{~m}, 3 \mathrm{H}), 2.15(\mathrm{~d}, J=10.8 \mathrm{~Hz}, 1 \mathrm{H})$, $1.98 \sim 1.73(\mathrm{~m}, 3 \mathrm{H}), 1.35(\mathrm{~s}, 3 \mathrm{H}) ;{ }^{13} \mathrm{C} \mathrm{NMR}(75 \mathrm{MHz}$, $\left.\mathrm{CDCl}_{3}\right) \delta: 202.4,191.2,172.9,170.5,152.6,146.0,129.7$, $118.5,89.3,76.0,65.6,61.6,60.8,52.4,48.9,48.0,40.3$, 36.7, 17.6, 12.0; EIMS m/z (\%) : $373\left(\mathrm{M}^{+}+1,4\right), 372$ $\left(\mathrm{M}^{+}, 18\right), 344$ (37), 340 (82), 326 (23), 312 (25), 294 (15), 285 (23), 268 (100), 267 (52), 251 (28), 239 (55), 218 (81), 213 (43), 195 (26), 185 (24), 171 (24), 157 (28), 141 (65), 128 (70), 115(87), 109 (25), 91 (23); HRMS calcd for $\mathrm{C}_{20} \mathrm{H}_{20} \mathrm{O}_{7} \mathrm{Na}(\mathrm{M}+\mathrm{Na})^{+}$395.1106, found 395.1103.

\section{2 .2 目标化合物 $11 \sim \mathbf{1 8}$ 的合成}

赤霉素衍生物 8 (98 mg, $0.25 \mathrm{mmol})$ 溶于无水的醇 (10 mL)中, 加入碳酸银(76 mg, $0.275 \mathrm{mmol}, 1.1$ equiv.), 搅拌回流 $24 \mathrm{~h}$. 减压蒸去溶剂, 残留物加入水 $(50 \mathrm{~mL})$, 用乙酸乙酯 $(15 \mathrm{~mL} \times 3)$ 萃取, 有机相用饱和食盐水 $(10$ $\mathrm{mL})$ 洗涤, 经无水硫酸钠干燥后, 过滤, 滤液经减压蒸 干所得粗品经硅胶柱层析纯化(200 300 目, 正己烷/乙 酸乙酯, $V: V=5: 1 \sim 2: 1)$, 得白色固体.

对映-3,15-二氧- $10 \beta$-羊基- $13 \beta$-甲氧基-20-失碳赤霉1,16-二烯-7,19-二羧酸 7-甲酯 19,10- 内酯(11): 产率 $33 \%$, m.p. $144 \sim 145{ }^{\circ} \mathrm{C} ;{ }^{1} \mathrm{H}$ NMR $\left(300 \mathrm{MHz}, \mathrm{CDCl}_{3}\right) \delta$ : 7.19 (d, $J=9.3 \mathrm{~Hz}, 1 \mathrm{H}), 6.17$ (s, 1H), 6.07 (d, $J=9.3 \mathrm{~Hz}$, 1H), 5.55 (s, 1H), $3.66(\mathrm{~d}, J=10.5 \mathrm{~Hz}, 1 \mathrm{H}), 3.64(\mathrm{~s}, 3 \mathrm{H})$, $3.29(\mathrm{~s}, 3 \mathrm{H}), 2.83(\mathrm{~d}, J=10.5 \mathrm{~Hz}, 1 \mathrm{H}), 2.42 \sim 2.10(\mathrm{~m}$, $5 \mathrm{H}), 1.92 \sim 1.62(\mathrm{~m}, 2 \mathrm{H}), 1.34(\mathrm{~s}, 3 \mathrm{H}) ;{ }^{13} \mathrm{C} \mathrm{NMR}(75 \mathrm{MHz}$, $\left.\mathrm{CDCl}_{3}\right) \delta: 202.5,191.2,173.0,170.5,149.4,146.0,129.7$, $119.3,89.3,80.8,65.6,61.6,60.6,52.4,50.8,49.2,48.5$, 34.6, 33.9, 17.7, 11.9; EIMS m/z (\%): $387\left(\mathrm{M}^{+}+1,6\right), 386$ $\left(\mathrm{M}^{+}, 18\right), 358$ (52), 355 (12), 342 (8), 331 (7), 313 (13), 310 (5), 299 (8), 282 (30), 281 (19), 255 (37), 237 (100), 223 (40), 217 (60), 205 (18), 196 (22), 185 (9), 175 (11), 165 (20), 153 (14), 141 (19), 128 (39), 115 (50), 109 (15); HRMS calcd. for $\mathrm{C}_{21} \mathrm{H}_{22} \mathrm{O}_{7} \mathrm{Na}(\mathrm{M}+\mathrm{Na})^{+} 409.1263$, found 409.1249 .

对映-3,15-二氧-13 $\beta$-乙氧基- $10 \beta$-羊基-20-失碳赤霉1,16-二烯-7,19-二羧酸 7-甲酯 19,10-内酯(12): 产率 $33 \%$, m.p. $123 \sim 125{ }^{\circ} \mathrm{C} ;{ }^{1} \mathrm{H}$ NMR $\left(300 \mathrm{MHz}, \mathrm{CDCl}_{3}\right) \delta$ : 7.20 (d, $J=9.3 \mathrm{~Hz}, 1 \mathrm{H}), 6.13$ (s, 1H), 6.06 (d, $J=9.3 \mathrm{~Hz}$, 1H), $5.54(\mathrm{~s}, 1 \mathrm{H}), 3.65(\mathrm{~d}, J=10.5 \mathrm{~Hz}, 1 \mathrm{H}), 3.63(\mathrm{~s}, 3 \mathrm{H})$, 
$3.53 \sim 3.34(\mathrm{~m}, 2 \mathrm{H}), 2.81(\mathrm{~d}, J=10.5 \mathrm{~Hz}, 1 \mathrm{H}), 2.42 \sim 2.20$ $(\mathrm{m}, 5 \mathrm{H}), 1.90 \sim 1.69(\mathrm{~m}, 2 \mathrm{H}), 1.32(\mathrm{~s}, 3 \mathrm{H}), 1.20(\mathrm{t}, J=7.2$ $\mathrm{Hz}, 3 \mathrm{H}) ;{ }^{13} \mathrm{C} \mathrm{NMR}\left(75 \mathrm{MHz}, \mathrm{CDCl}_{3}\right) \delta: 202.6,191.2$, $173.0,170.6,149.9,146.1,129.6,118.9,89.3,80.4,65.5$, $61.5,60.6,58.6,52.3,49.2,48.5,35.3,34.6,17.6,15.8$, 11.9; EIMS $m / z(\%): 401\left(\mathrm{M}^{+}+1,2\right), 400\left(\mathrm{M}^{+}, 11\right), 372$ (35), 356 (9), 345 (68), 327 (13), 313 (8), 296 (16), 269 (27), 267 (29), 251 (100), 231 (36), 223 (32), 208 (27), 196 (32), 185 (18), 178 (15), 157 (12), 149 (15), 141 (17), 128 (32), 115 (50), 109 (17); HRMS calcd for $\mathrm{C}_{22} \mathrm{H}_{24} \mathrm{O}_{7} \mathrm{Na}$ $(\mathrm{M}+\mathrm{Na})^{+}$423.1419, found 423.1403 .

对映-3,15-二氧- $13 \beta$-羊基- $13 \beta$-异丙氧基-20-失碳赤 霉-1,16-二烯-7,19-二羧酸 7-甲酯 19,10-内酯(13): 产率 29\%, m.p. $112 \sim 114{ }^{\circ} \mathrm{C}$; ${ }^{1} \mathrm{H}$ NMR $\left(300 \mathrm{MHz}, \mathrm{CDCl}_{3}\right) \delta$ : 7.19 (d, $J=9.3 \mathrm{~Hz}, 1 \mathrm{H}), 6.12(\mathrm{~s}, 1 \mathrm{H}), 6.07$ (d, $J=9.3 \mathrm{~Hz}$, $1 \mathrm{H}), 5.61(\mathrm{~s}, 1 \mathrm{H}), 3.82 \sim 3.70(\mathrm{~m}, 1 \mathrm{H}), 3.65(\mathrm{~s}, 3 \mathrm{H}), 3.63$ $(\mathrm{d}, J=10.2 \mathrm{~Hz}, 1 \mathrm{H}), 2.82$ (d, $J=10.2 \mathrm{~Hz}, 1 \mathrm{H}), 2.44$ (d, $J=$ $10.8 \mathrm{~Hz}, 1 \mathrm{H}), 2.41 \sim 2.15(\mathrm{~m}, 4 \mathrm{H}), 1.94 \sim 1.70(\mathrm{~m}, 2 \mathrm{H})$, 1.34 (s, 3H), $1.20(\mathrm{~d}, J=5.7 \mathrm{~Hz}, 3 \mathrm{H}), 1.18(\mathrm{~d}, J=5.7 \mathrm{~Hz}$, $3 \mathrm{H}) ;{ }^{13} \mathrm{C}$ NMR $\left(75 \mathrm{MHz}, \mathrm{CDCl}_{3}\right) \delta: 202.8,191.2,172.9$, $170.6,151.1,146.0,129.6,118.9,89.3,80.5,66.0,65.5$, $61.5,60.5,52.3,49.3,48.5,36.3,36.1,24.9,24.6,17.7$, 11.9; EIMS $m / z(\%): 415\left(\mathrm{M}^{+}+1,9\right), 414\left(\mathrm{M}^{+}, 35\right), 386$ (35), 372 (25), 370 (16), 358 (9), 344 (64), 340 (21), 327 (25), 325 (33), 312 (38), 310 (35), 299 (34), 296 (17), 269 (33), 265 (17), 251 (28), 239 (100), 223 (70), 213 (51), 211 (42), 196 (47), 189 (92), 185 (81), 171 (69), 165 (56), 157 (42), 153 (26), 141 (68), 128 (26), 115 (17); HRMS calcd for $\mathrm{C}_{23} \mathrm{H}_{26} \mathrm{O}_{7} \mathrm{Na}(\mathrm{M}+\mathrm{Na})^{+} 437.1576$, found 437.1588 .

对映-3,15-二氧- $10 \beta$-羊基- $13 \beta$-丙氧基- 20 -失碳赤霉1,16-二烯-7,19-二羧酸 7-甲酯 19,10-内酯(14): 产率 88\%, m.p. 120 121 ${ }^{\circ} \mathrm{C} ;{ }^{1} \mathrm{H}$ NMR (300 MHz, $\left.\mathrm{CDCl}_{3}\right) \delta$ : 7.19 (d, $J=9.3 \mathrm{~Hz}, 1 \mathrm{H}), 6.11(\mathrm{~s}, 1 \mathrm{H}), 6.04$ (d, $J=9.3 \mathrm{~Hz}$, $1 \mathrm{H}), 5.53$ (s, 1H), $3.62(\mathrm{~s}, 3 \mathrm{H}), 3.62(\mathrm{~d}, J=10.5 \mathrm{~Hz}, 1 \mathrm{H})$, $3.52 \sim 3.22(\mathrm{~m}, 2 \mathrm{H}), 2.80(\mathrm{~d}, J=10.5 \mathrm{~Hz}, 1 \mathrm{H}), 2.40 \sim 2.10$ $(\mathrm{m}, 5 \mathrm{H}), 1.92 \sim 1.67(\mathrm{~m}, 2 \mathrm{H}), 1.65 \sim 1.52(\mathrm{~m}, 2 \mathrm{H}), 1.31(\mathrm{~s}$, $3 \mathrm{H}), 0.93(\mathrm{t}, J=7.2 \mathrm{~Hz}, 3 \mathrm{H}) ;{ }^{13} \mathrm{C} \mathrm{NMR}\left(75 \mathrm{MHz}, \mathrm{CDCl}_{3}\right)$ $\delta: 202.7,191.3,173.0,170.6,150.0,146.1,129.6,119.0$, $89.4,80.2,65.5,64.7,61.5,60.6,52.3,49.2,48.5,35.1$, 34.6, 23.5, 17.6, 11.9, 10.7; EIMS $m / z(\%): 415\left(\mathrm{M}^{+}+1\right.$, 7), $414\left(\mathrm{M}^{+}, 16\right), 386(29), 370$ (13), 355 (8), 341 (23), 327 (14), 325 (25), 311 (51), 310 (57), 299 (22), 283 (38), 269 (24), 265 (100), 251 (31), 241 (45), 239 (49), 223 (55), 213 (46), 211 (38), 196 (50), 185 (36), 171 (22), 165 (15), 157 (10), 141 (15), 128 (32), 115 (41); HRMS calcd for
$\mathrm{C}_{23} \mathrm{H}_{26} \mathrm{O}_{7} \mathrm{Na}(\mathrm{M}+\mathrm{Na})^{+} 437.1576$, found 437.1584 .

对映 $13 \beta$-丁氧基-3,15-二氧- $10 \beta$-羟基-20-失碳赤霉1,16-二烯-7,19-二羧酸 7-甲酯 19,10- 内酯(15): 产率 29\%, m.p. 115 116 ${ }^{\circ} \mathrm{C} ;{ }^{1} \mathrm{H}$ NMR $\left(300 \mathrm{MHz}, \mathrm{CDCl}_{3}\right) \delta$ : 7.19 (d, $J=9.3 \mathrm{~Hz}, 1 \mathrm{H}), 6.13$ (s, 1H), 6.06 (d, $J=9.3 \mathrm{~Hz}$, $1 \mathrm{H}), 5.54$ (s, 1H), 3.64 (s, 3H), 3.63 (d, $J=10.2 \mathrm{~Hz}, 1 \mathrm{H})$, $3.48 \sim 3.30(\mathrm{~m}, 2 \mathrm{H}), 2.81(\mathrm{~d}, J=10.2 \mathrm{~Hz}, 1 \mathrm{H}), 2.41 \sim 2.18$ $(\mathrm{m}, 5 \mathrm{H}), 1.92 \sim 1.69(\mathrm{~m}, 2 \mathrm{H}), 1.62 \sim 1.52(\mathrm{~m}, 2 \mathrm{H}), 1.48 \sim$ $1.34(\mathrm{~m}, 2 \mathrm{H}), 1.24(\mathrm{~s}, 3 \mathrm{H}), 0.93(\mathrm{t}, J=7.2 \mathrm{~Hz}, 3 \mathrm{H}) ;{ }^{13} \mathrm{C}$ NMR (75 MHz, $\left.\mathrm{CDCl}_{3}\right) \delta: 202.7,191.2,172.9,170.6$, $150.1,146.1,129.6,118.9,89.3,80.2,65.6,62.9,61.6$, 60.6, 52.3, 49.3, 48.6, 35.1, 34.7, 32.4, 19.5, 17.7, 14.0, 11.9; EIMS $m / z(\%): 430\left(\mathrm{M}^{+}+2,6\right), 428\left(\mathrm{M}^{+}, 18\right), 402$ (5), 400 (19), 384 (20), 358 (28), 355 (29), 340 (10), 325 (59), 324 (63), 311 (41), 299 (34), 285 (31), 279 (100), 267 (49), 251 (65), 239 (69), 225 (33), 223 (92), 222 (51), 213 (45), 196 (85), 185 (43), 178 (27), 171 (27), 165 (17), 158 (15), 141 (14), 128 (30), 115 (42), 97 (17); HRMS calcd for $\mathrm{C}_{24} \mathrm{H}_{28} \mathrm{O}_{7} \mathrm{Na}(\mathrm{M}+\mathrm{Na})^{+}$451.1732, found 451.1737.

对映-3,15-二氧- $10 \beta$-羊基- $13 \beta$-异丁氧基-20-失碳赤 霉-1,16-二烯-7,19-二羧酸 7-甲酯 19,10-内酯(16): 产率 39\%, m.p. 92 93 ${ }^{\circ} \mathrm{C} ;{ }^{1} \mathrm{H}$ NMR (300 MHz, $\left.\mathrm{CDCl}_{3}\right) \delta$ : 7.19 (d, $J=9.3 \mathrm{~Hz}, 1 \mathrm{H}), 6.12$ (s, 1H), 6.06 (d, $J=9.3 \mathrm{~Hz}$, $1 \mathrm{H}), 5.54(\mathrm{~s}, 1 \mathrm{H}), 3.64(\mathrm{~s}, 3 \mathrm{H}), 3.63(\mathrm{~d}, J=10.5 \mathrm{~Hz}, 1 \mathrm{H})$, $3.22 \sim 3.08(\mathrm{~m}, 2 \mathrm{H}), 2.81(\mathrm{~d}, J=10.5 \mathrm{~Hz}, 1 \mathrm{H}), 2.42 \sim 2.18$ $(\mathrm{m}, 5 \mathrm{H}), 1.92 \sim 1.65(\mathrm{~m}, 3 \mathrm{H}), 1.33(\mathrm{~s}, 3 \mathrm{H}), 0.94(\mathrm{~d}, J=6.7$ $\mathrm{Hz}, 3 \mathrm{H}), 0.93(\mathrm{~d}, J=6.7 \mathrm{~Hz}, 3 \mathrm{H}) ;{ }^{13} \mathrm{C} \mathrm{NMR}(75 \mathrm{MHz}$, $\left.\mathrm{CDCl}_{3}\right) \delta: 202.8,191.3,173.0,170.6,150.2,146.1,129.6$, $119.0,89.4,80.1,69.7,65.6,61.5,60.6,52.4,49.3,48.5$, 35.0, 34.7, 29.0, 19.5, 17.7, 11.9; EIMS $m / z(\%): 428\left(\mathrm{M}^{+}\right.$, 7), 400 (8), 384 (6), 373 (22), 355 (9), 341 (14), 325 (16), 313 (15), 295 (33), 283 (14), 279 (51), 269 (100), 267 (42), 253 (12), 241 (78), 239 (52), 223 (40), 211 (18), 195 (21), 185 (27), 171 (19), 165 (23), 150 917), 141 (28), 128 (17), 115 (12); HRMS calcd for $\mathrm{C}_{24} \mathrm{H}_{28} \mathrm{O}_{7} \mathrm{Na}(\mathrm{M}+\mathrm{Na})^{+}$ 451.1732, found 451.1739 .

对映-3,15-二氧- $10 \beta$-羊基- $13 \beta$-戊氧基-20-失碳赤霉1,16-二烯-7,19-二羧酸 7-甲酯 19,10- 内酯(17): 产率 42\%, m.p. 98 100 ${ }^{\circ} \mathrm{C}$; ${ }^{1} \mathrm{H}$ NMR $\left(300 \mathrm{MHz}, \mathrm{CDCl}_{3}\right) \delta$ : 7.18 ( d, $J=9.3 \mathrm{~Hz}, 1 \mathrm{H}), 6.07$ (s, 1H), 6.01 (d, $J=9.3 \mathrm{~Hz}$, $1 \mathrm{H}), 5.49(\mathrm{~s}, 1 \mathrm{H}), 3.58(\mathrm{~s}, 3 \mathrm{H}), 3.56(\mathrm{~d}, J=10.5 \mathrm{~Hz}, 1 \mathrm{H})$, $3.42 \sim 3.23(\mathrm{~m}, 2 \mathrm{H}), 2.77(\mathrm{~d}, J=10.5 \mathrm{~Hz}, 1 \mathrm{H}), 2.38 \sim 2.11$ $(\mathrm{m}, 5 \mathrm{H}), 1.92 \sim 1.62(\mathrm{~m}, 2 \mathrm{H}), 1.61 \sim 1.46(\mathrm{~m}, 2 \mathrm{H}), 1.35 \sim$ $1.22(\mathrm{~m}, 4 \mathrm{H}), 1.27(\mathrm{~s}, 3 \mathrm{H}), 0.84(\mathrm{t}, J=6.7 \mathrm{~Hz}, 3 \mathrm{H}) ;{ }^{13} \mathrm{C}$ NMR (75 MHz, $\left.\mathrm{CDCl}_{3}\right) \delta: 202.6,191.2,172.9,170.5$, 
$149.9,146.1,129.4,118.8,89.3,80.1,65.4,63.0,61.4$, $60.4,52.2,49.0,48.3,35.0,34.4,29.9,28.3,22.5,17.5$, 14.0, 11.8; EIMS $m / z(\%): 443\left(\mathrm{M}^{+}+1,5\right), 442\left(\mathrm{M}^{+}, 22\right)$, 414 (23), 398 (10), 386 (6), 369 (12), 355 (5), 338 (19), 325 (17), 311 (13), 293 (100), 285 (12), 269 (47), 251 (35), 241 (68), 239 (41), 223 (37), 213 (34), 203 (16), 196 (20), 185 (25), 171 (23), 157 (12), 150 (17), 141 (22), 128 (13), 109 (4); HRMS calcd for $\mathrm{C}_{25} \mathrm{H}_{30} \mathrm{O}_{7} \mathrm{Na}(\mathrm{M}+\mathrm{Na})^{+}$ 465.1889, found 465.1900 .

对映-3,15-二氧- $10 \beta$-羊基-13 $\beta$-异戊氧基-20-失碳赤 需-1,16-二烯-7,19-二羧酸 7-甲酯 19,10-内酯(18): 产率 12\%, m.p. 90 92 ${ }^{\circ} \mathrm{C} ;{ }^{1} \mathrm{H}$ NMR $\left(300 \mathrm{MHz}, \mathrm{CDCl}_{3}\right) \delta$ : $7.15(\mathrm{~d}, J=9.3 \mathrm{~Hz}, 1 \mathrm{H}), 6.07$ (s, 1H), 6.01 (d, $J=9.3 \mathrm{~Hz}$, 1H), 5.48 (s, 1H), 3.58 (s, 3H), 3.57 (d, $J=10.5 \mathrm{~Hz}, 1 \mathrm{H})$, $3.45 \sim 3.23(\mathrm{~m}, 2 \mathrm{H}), 2.76(\mathrm{~d}, J=10.5 \mathrm{~Hz}, 1 \mathrm{H}), 2.38 \sim 2.10$ $(\mathrm{m}, 5 \mathrm{H}), 1.92 \sim 1.62(\mathrm{~m}, 3 \mathrm{H}), 1.50 \sim 1.38(\mathrm{~m}, 2 \mathrm{H}), 1.27(\mathrm{~s}$, $3 \mathrm{H}), 0.85(\mathrm{~d}, J=6.7 \mathrm{~Hz}, 3 \mathrm{H}), 0.84(\mathrm{~d}, J=6.7 \mathrm{~Hz}, 3 \mathrm{H}) ;{ }^{13} \mathrm{C}$ NMR (75 MHz, $\left.\mathrm{CDCl}_{3}\right) \delta: 202.7,191.3,173.0,170.6$, $150.2,146.1,129.6,119.0,89.4,80.2,65.6,61.6,61.5$, $60.6,52.4,49.3,48.5,39.2,35.0,34.7,25.1,22.8,22.7$, 17.7, 11.9; EIMS $m / z(\%): 443\left(\mathrm{M}^{+}+1,3\right), 442\left(\mathrm{M}^{+}, 0.9\right)$, 415 (3), 398 (7), 387 (3), 373 (5), 355 (7), 341 (14), 338 (15), 325 (20), 313 (19), 293 (50), 285 (17), 269 (88), 261 (17), 251 (40), 241 (85), 239 (57), 232 (19), 223 (42), 213 (39), 211 (35), 196 (32), 185 (36), 171 (52), 158 (27), 150 (29), 141 (35), 129 (27), 115 (12), 106 (10), 91 (7), 82 (18), 70 100); HRMS calcd for $\mathrm{C}_{25} \mathrm{H}_{30} \mathrm{O}_{7} \mathrm{Na}(\mathrm{M}+\mathrm{Na})^{+}$ 465.1889 , found 465.1892 .

对映-13 $\beta, 10 \beta$-二羟基-3,15-二氧-20-失碳赤霉-1,16二烯-7,19-二羧酸 7-苯甲酯 19,10-内酯(19): 赤霉素衍 生物 9 (467 mg, $1.0 \mathrm{mmol})$ 溶于丙酮 $(8 \mathrm{~mL})$ 和水 $(2 \mathrm{~mL})$ 中, 加入碳酸银(276 mg, $1.0 \mathrm{mmol}, 1.0$ equiv.), 搅拌回流 24 h. 减压蒸去溶剂, 残留物加入水 $(50 \mathrm{~mL})$, 用乙酸乙酯 $(15 \mathrm{~mL} \times 3)$ 萃取, 经无水硫酸钠干燥后, 过滤, 滤液经 减压蒸干所得粗品经硅胶柱层析纯化(200 300 目, 正 己烷/乙酸乙酯, $V: V=5: 1 \sim 2: 1)$, 得白色针状固体 (253 mg, 54.6\%). m.p. 216 217 ${ }^{\circ} \mathrm{C}$; ${ }^{1} \mathrm{H}$ NMR (300 MHz, $\left.\mathrm{CDCl}_{3}\right) \delta: 7.38 \sim 7.16(\mathrm{~m}, 6 \mathrm{H}), 6.08(\mathrm{~d}, J=9.6 \mathrm{~Hz}, 1 \mathrm{H})$, $6.06(\mathrm{~s}, 1 \mathrm{H}), 5.65(\mathrm{~s}, 1 \mathrm{H}), 5.06(\mathrm{~s}, 2 \mathrm{H}), 3.67(\mathrm{~d}, J=10.5$ $\mathrm{Hz}, 1 \mathrm{H}), 2.86$ (d, $J=10.5 \mathrm{~Hz}, 1 \mathrm{H}), 2.51$ (d, $J=11.1 \mathrm{~Hz}$, $1 \mathrm{H}), 2.32 \sim 2.14(\mathrm{~m}, 3 \mathrm{H}), 2.07(\mathrm{~d}, J=11.1 \mathrm{~Hz}, 1 \mathrm{H}), 1.97 \sim$ $1.80(\mathrm{~m}, 3 \mathrm{H}), 1.65 \sim 1.55(\mathrm{~m}, 1 \mathrm{H}), 1.33(\mathrm{~s}, 3 \mathrm{H}) ;{ }^{13} \mathrm{C} \mathrm{NMR}$ $\left(75 \mathrm{MHz}, \mathrm{CDCl}_{3}\right) \delta: 202.2,191.2,172.9,170.0,152.8$, $146.0,135.1,129.7,128.8,128.7,128.6,118.1,89.3,75.9$, $67.5,65.5,61.6,60.8,49.1,48.1,40.4,36.5,17.5,12.0$;
EIMS $m / z$ (\%): $448\left(\mathrm{M}^{+}, 2\right), 420$ (3), 357 (3), 342 (18), 313 (8), 295 (5), 280 (16), 267 (21), 249 (7), 221 (16), 213 (4), 195 (5), 171 (4), 151 (13), 148 (20), 128 (12), 115 (12), 107 (26), 91 (100); HRMS calcd for $\mathrm{C}_{26} \mathrm{H}_{24} \mathrm{O}_{7} \mathrm{Na}$ $(\mathrm{M}+\mathrm{Na})^{+}$471.1419, found 471.1408 .

\subsection{3 目标化合物 $20 \sim 27$ 的合成}

赤霉素衍生物 9 (299 mg, $0.64 \mathrm{mmol})$ 溶于无水的醇 (15 mL)中, 加入碳酸银(212 mg, $0.77 \mathrm{mmol}, 1.2$ equiv.), 搅拌回流 $24 \mathrm{~h}$. 减压蒸去溶剂, 残留物加入水 $(70 \mathrm{~mL})$, 用乙酸乙酯 $(20 \mathrm{~mL} \times 2)$ 萃取, 有机相用饱和食盐水 $(10$ $\mathrm{mL})$ 洗涤, 经无水硫酸钠干燥后, 过滤, 滤液经减压蒸 干所得粗品经硅胶柱层析纯化(200３00 目，正己烷/乙 酸乙酯, $V: V=5: 1 \sim 2: 1$ ), 得白色固体.

对映-3,15-二氧- $10 \beta$-羊基- $13 \beta$-甲氧基-20-失碳赤霉1,16-二烯-7,19-二羧酸 7-苯甲酯 19,10-内酯(20): 产率 25\%, m.p. 201 202 ${ }^{\circ} \mathrm{C} ;{ }^{1} \mathrm{H}$ NMR $\left(300 \mathrm{MHz}, \mathrm{CDCl}_{3}\right) \delta$ : $7.32 \sim 7.21(\mathrm{~m}, 5 \mathrm{H}), 7.19(\mathrm{~d}, J=9.3 \mathrm{~Hz}, 1 \mathrm{H}), 6.14(\mathrm{~s}, 1 \mathrm{H})$, 6.07 (d, $J=9.3 \mathrm{~Hz}, 1 \mathrm{H}), 5.49$ (s, 1H), 5.08 (d, $J=12.0 \mathrm{~Hz}$, $1 \mathrm{H}), 5.00(\mathrm{~d}, J=12.0 \mathrm{~Hz}, 1 \mathrm{H}), 3.66(\mathrm{~d}, J=10.5 \mathrm{~Hz}, 1 \mathrm{H})$, 3.09 (s, 3H), 2.87 (d, $J=10.5 \mathrm{~Hz}, 1 \mathrm{H}), 2.37 \sim 2.20(\mathrm{~m}$, $4 \mathrm{H}), 1.92 \sim 1.68(\mathrm{~m}, 2 \mathrm{H}), 1.35(\mathrm{~s}, 3 \mathrm{H}) ;{ }^{13} \mathrm{C} \mathrm{NMR}(75 \mathrm{MHz}$, $\left.\mathrm{CDCl}_{3}\right) \delta: 202.6,191.2,172.9,169.9,148.8,146.0,135.0$, $129.7,128.8,128.6,128.6,119.3,89.3,81.0,67.5,65.5$, $61.5,60.6,50.5,49.3,48.6,35.6,33.3,17.6,12.0$; EIMS m/z (\%): $462\left(\mathrm{M}^{+}, 0.5\right), 418$ (1), 389 (2), 371 (15), 356 (6), 343 (19), 327 (4), 312 (4), 280 (6), 269 (13), 237 (5), 221 (15), 217 (7), 195 (5), 165 (11), 149 (10), 128 (7), 115 (12), 91 (100); HRMS calcd for $\mathrm{C}_{27} \mathrm{H}_{26} \mathrm{O}_{7} \mathrm{Na}(\mathrm{M}+\mathrm{Na})^{+}$ 485.1576, found 485.1561.

对映-3,15-二氧- $13 \beta$-乙氧基- $10 \beta$-羊基-20-失碳赤霉1,16-二烯-7,19-二羧酸 7-苯甲酯 19,10-内酯(21): 产率 25\%, m.p. $220 \sim 222{ }^{\circ} \mathrm{C} ;{ }^{1} \mathrm{H}$ NMR (300 MHz, $\left.\mathrm{CDCl}_{3}\right) \delta$ : $7.37 \sim 7.21(\mathrm{~m}, 5 \mathrm{H}), 7.19(\mathrm{~d}, J=9.3 \mathrm{~Hz}, 1 \mathrm{H}), 6.12(\mathrm{~s}, 1 \mathrm{H})$, 6.07 (d, $J=9.3 \mathrm{~Hz}, 1 \mathrm{H}), 5.49$ (s, 1H), 5.08 (d, $J=12.0 \mathrm{~Hz}$, $1 \mathrm{H}), 5.00(\mathrm{~d}, J=12.0 \mathrm{~Hz}, 1 \mathrm{H}), 3.65(\mathrm{~d}, J=10.5 \mathrm{~Hz}, 1 \mathrm{H})$, $3.32 \sim 3.08(\mathrm{~m}, 2 \mathrm{H}), 2.85(\mathrm{~d}, J=10.5 \mathrm{~Hz}, 1 \mathrm{H}), 2.35 \sim 2.17$ $(\mathrm{m}, 5 \mathrm{H}), 1.92 \sim 1.71(\mathrm{~m}, 2 \mathrm{H}), 1.35(\mathrm{~s}, 3 \mathrm{H}), 1.15(\mathrm{t}, J=6.9$ $\mathrm{Hz}, 3 \mathrm{H}) ;{ }^{13} \mathrm{C} \mathrm{NMR}\left(75 \mathrm{MHz}, \mathrm{CDCl}_{3}\right) \delta: 202.7,191.2$, $172.9,169.9,149.5,146.1,135.1,129.6,128.8,128.6$, $118.9,89.3,80.6,67.5,65.5,61.5,60.7,58.4,49.3,48.6$, 36.2, 34.1, 17.6, 15.8, 14.3, 12.0; EIMS m/z (\%): $476\left(\mathrm{M}^{+}\right.$, 1), 448 (0.2), 432 (0.8), 421 (2), 385 (8), 357 (15), 341 (6), 324 (4), 311 (5), 283 (9), 267 (10), 255 (3), 231 (5), 221 (11), 213 (4), 195 (5), 179 (7), 149 (8), 128 (6), 115 (8), 107 (5), 91 (100); HRMS calcd for $\mathrm{C}_{28} \mathrm{H}_{28} \mathrm{O}_{7} \mathrm{Na}(\mathrm{M}+\mathrm{Na})$ 
499.1732, found 499.1724.

对映-3,15-二氧- $10 \beta$-羊基- $13 \beta$-异丙氧基- 20 -失碳赤 霉-1,16-二烯-7,19-二羧酸 7-苯甲酯 19,10-内酯(22): 产 率 29\%, m.p. $168 \sim 169{ }^{\circ} \mathrm{C} ;{ }^{1} \mathrm{H}$ NMR $\left(300 \mathrm{MHz}, \mathrm{CDCl}_{3}\right)$ $\delta: 7.27 \sim 7.13(\mathrm{~m}, 5 \mathrm{H}), 7.12(\mathrm{~d}, J=9.3 \mathrm{~Hz}, 1 \mathrm{H}), 6.03$ (s, 1H), 5.99 (d, $J=9.3 \mathrm{~Hz}, 1 \mathrm{H}), 5.49$ (s, 1H), 5.00 (d, $J=$ $11.7 \mathrm{~Hz}, 1 \mathrm{H}), 4.90$ (d, $J=11.7 \mathrm{~Hz}, 1 \mathrm{H}), 3.56$ (d, $J=10.5$ $\mathrm{Hz}, 1 \mathrm{H}), 3.33 \sim 3.23(\mathrm{~m}, 1 \mathrm{H}), 2.79(\mathrm{~d}, J=10.5 \mathrm{~Hz}, 1 \mathrm{H})$, $2.30 \sim 2.10(\mathrm{~m}, 5 \mathrm{H}), 1.81 \sim 1.63(\mathrm{~m}, 2 \mathrm{H}), 1.28(\mathrm{~s}, 3 \mathrm{H})$, $1.01(\mathrm{~d}, J=6.3 \mathrm{~Hz}, 3 \mathrm{H}), 0.98(\mathrm{~d}, J=6.3 \mathrm{~Hz}, 3 \mathrm{H}) ;{ }^{13} \mathrm{C}$ NMR (75 MHz, $\left.\mathrm{CDCl}_{3}\right) \delta: 202.8,191.2,172.9,169.9$, $150.6,146.1,135.0,129.6,128.9,128.5,119.1,89.3,80.7$, $67.5,65.9,65.5,61.4,60.5,49.2,48.4,37.0,35.7,24.8$, 24.4, 17.6, 12.0; EIMS m/z (\%): $490\left(\mathrm{M}^{+}, 4\right), 462$ (1), 399 (1), 384 (2), 375 (8), 357 (17), 329 (24), 313 (22), 311 (20), 295 (7), 285 (8), 267 (15), 239 (10), 221 (9), 213 (9), 211 (7), 185 (10), 171 (13), 165 (6), 149 (12), 128 (3), 91 (100); HRMS calcd for $\mathrm{C}_{29} \mathrm{H}_{30} \mathrm{O}_{7} \mathrm{Na}(\mathrm{M}+\mathrm{Na})^{+}$513.1889, found 513.1894 .

对映-3,15-二氧- $10 \beta$-羊基- $13 \beta$-丙氧基- 20 -失碳赤霉1,16-二烯-7,19-二羧酸 7-苯甲酯 19,10-内酯 $(23)$ : 产率 58\%, m.p. 210 211 ${ }^{\circ} \mathrm{C} ;{ }^{1} \mathrm{H}$ NMR (300 MHz, $\left.\mathrm{CDCl}_{3}\right) \delta$ : $7.33 \sim 7.20(\mathrm{~m}, 5 \mathrm{H}), 7.20(\mathrm{~d}, J=9.3 \mathrm{~Hz}, 1 \mathrm{H}), 6.12(\mathrm{~s}, 1 \mathrm{H})$, 6.07 (d, $J=9.3 \mathrm{~Hz}, 1 \mathrm{H}), 5.49$ (s, 1H), 5.08 (d, $J=11.7 \mathrm{~Hz}$, $1 \mathrm{H}), 4.99$ (d, $J=11.7 \mathrm{~Hz}, 1 \mathrm{H}), 3.65$ (d, $J=10.5 \mathrm{~Hz}, 1 \mathrm{H})$, $3.19 \sim 3.00(\mathrm{~m}, 2 \mathrm{H}), 2.86(\mathrm{~d}, J=10.5 \mathrm{~Hz}, 1 \mathrm{H}), 2.38 \sim 2.18$ $(\mathrm{m}, 5 \mathrm{H}), 1.92 \sim 1.73(\mathrm{~m}, 2 \mathrm{H}), 1.61 \sim 1.47(\mathrm{~m}, 2 \mathrm{H}), 1.35(\mathrm{~s}$, $3 \mathrm{H}), 0.89$ (d, $J=7.5 \mathrm{~Hz}, 3 \mathrm{H}) ;{ }^{13} \mathrm{C}$ NMR $\left(75 \mathrm{MHz}, \mathrm{CDCl}_{3}\right.$ ) $\delta: 202.7,191.2,172.9,169.9,149.5,146.1,135.0,129.6$, $128.8,128.6,119.0,89.3,80.4,67.5,65.5,64.6,61.5,60.6$, 49.2, 48.6, 36.1, 34.0, 23.5, 17.6, 12.0, 10.7; EIMS m/z (\%): $490\left(\mathrm{M}^{+}, 1\right), 463$ (0.8), 446 (1), 435 (0.9), 399 (2), 373 (3), 371 (4), 357 (8), 339 (4), 329 (5), 313 (3), 311 (11), 297 (9), 285 (4), 267 (13), 239 (4), 221 (10), 213 (5), 193 (5), 149 (3), 128 (3), 115 (4), 107 (5), 91 (100); HRMS calcd for $\mathrm{C}_{29} \mathrm{H}_{30} \mathrm{O}_{7} \mathrm{Na}(\mathrm{M}+\mathrm{Na})^{+} 513.1889$, found 513.1892 .

对映-13 $\beta$-丁氧基-3,15-二氧- $10 \beta$-羟基- 20 -失碳赤霉1,16-二烯-7,19-二羧酸 7-苯甲酯 19,10-内酯(24): 产率 $23 \%$, m.p. 197 $198{ }^{\circ} \mathrm{C} ;{ }^{1} \mathrm{H}$ NMR (300 MHz, $\left.\mathrm{CDCl}_{3}\right) \delta$ : $7.37 \sim 7.18(\mathrm{~m}, 5 \mathrm{H}), 7.18(\mathrm{~d}, J=9.3 \mathrm{~Hz}, 1 \mathrm{H}), 6.11(\mathrm{~s}, 1 \mathrm{H})$, 6.07 (d, $J=9.3 \mathrm{~Hz}, 1 \mathrm{H}), 5.48(\mathrm{~s}, 1 \mathrm{H}), 5.07$ (d, $J=11.8 \mathrm{~Hz}$, $1 \mathrm{H}), 4.98$ (d, $J=11.8 \mathrm{~Hz}, 1 \mathrm{H}), 3.65$ (d, $J=10.5 \mathrm{~Hz}, 1 \mathrm{H})$, $3.22 \sim 3.02(\mathrm{~m}, 2 \mathrm{H}), 2.86(\mathrm{~d}, J=10.5 \mathrm{~Hz}, 1 \mathrm{H}), 2.33 \sim 2.10$ $(\mathrm{m}, 6 \mathrm{H}), 1.93 \sim 1.70(\mathrm{~m}, 3 \mathrm{H}), 1.61 \sim 1.45(\mathrm{~m}, 2 \mathrm{H}), 1.35(\mathrm{~s}$,
$3 \mathrm{H}), 0.90(\mathrm{~d}, J=7.3 \mathrm{~Hz}, 3 \mathrm{H}) ;{ }^{13} \mathrm{C} \mathrm{NMR}\left(75 \mathrm{MHz}, \mathrm{CDCl}_{3}\right)$ $\delta: 202.7,191.2,172.9,169.9,149.5,146.1,135.0,129.6$, $128.8,128.5,119.0,89.3,80.4,67.5,65.5,62.7,61.4,60.6$, $49.2, \quad 48.6,36.0,34.0,32.3,19.4,17.6,14.0,12.0$; EIMS $m / z(\%): 504\left(\mathrm{M}^{+}, 0.9\right), 460$ (1), 449 (2), 431 (1.5), 385 (4), 360 (7), 357 (10), 339 (4), 329 (6), 311 (7), 280 (3), 267 (7), 255 (6), 239 (5), 221 (5), 195 (5), 171 (4), 165 (3), 128 (3), 107 (5), 91 (100); HRMS calcd for $\mathrm{C}_{30} \mathrm{H}_{33} \mathrm{O}_{7}$ $(\mathrm{M}+\mathrm{H})^{+}$505.2226, found 505.2194.

对映-3,15-二氧- $10 \beta$-羊基- $13 \beta$-异丁氧基-20-失碳赤 霉-1,16-二烯-7,19-二羧酸 7-苯甲酯 19,10-内酯(25): 产 率 $72 \%$, m.p. $160 \sim 161{ }^{\circ} \mathrm{C} ;{ }^{1} \mathrm{H}$ NMR $\left(300 \mathrm{MHz}, \mathrm{CDCl}_{3}\right)$ $\delta: 7.27 \sim 7.10(\mathrm{~m}, 5 \mathrm{H}), 7.11(\mathrm{~d}, J=9.3 \mathrm{~Hz}, 1 \mathrm{H}), 6.03(\mathrm{~s}$, $1 \mathrm{H}), 5.99$ (d, $J=9.3 \mathrm{~Hz}, 1 \mathrm{H}), 5.40$ (s, 1H), 5.00 (d, $J=$ $11.8 \mathrm{~Hz}, 1 \mathrm{H}), 4.90$ (d, $J=11.8 \mathrm{~Hz}, 1 \mathrm{H}), 3.57$ (d, $J=10.5$ $\mathrm{Hz}, 1 \mathrm{H}), 2.94 \sim 2.72(\mathrm{~m}, 2 \mathrm{H}), 2.78(\mathrm{~d}, J=10.5 \mathrm{~Hz}, 1 \mathrm{H})$, $2.30 \sim 2.05(\mathrm{~m}, 5 \mathrm{H}), 1.83 \sim 1.59(\mathrm{~m}, 3 \mathrm{H}), 1.27(\mathrm{~s}, 3 \mathrm{H})$, $0.81(\mathrm{~d}, J=6.5 \mathrm{~Hz}, 3 \mathrm{H}), 0.80(\mathrm{~d}, J=6.5 \mathrm{~Hz}, 3 \mathrm{H}) ;{ }^{13} \mathrm{C}$ NMR (75 MHz, $\left.\mathrm{CDCl}_{3}\right) \delta: 202.8,191.2,172.9,169.9$, $149.6,146.1,135.0,129.6,128.7,128.6,119.1,89.3,80.3$, $69.5,67.5,65.5,61.4,60.6,49.2,48.6,35.9,34.1,28.9$, 19.5, 19.4, 17.5, 12.0; TOF-MS $m / z(\%): 505\left(\mathrm{M}^{+}+1\right.$, 100\%), 483 (55), 397 (28), 325 (25), 301 (14), 149 (20); HRMS calcd for $\mathrm{C}_{30} \mathrm{H}_{33} \mathrm{O}_{7}(\mathrm{M}+\mathrm{H})^{+}$505.2226, found 505.2195 .

对映-3,15-二氧- $10 \beta$-羊基- $13 \beta$-戊氧基-20-失碳赤霉1,16-二烯-7,19-二羧酸 7-苯甲酯 19,10-内酯(26): 产率 46\%, m.p. 120 122 ${ }^{\circ} \mathrm{C} ;{ }^{1} \mathrm{H}$ NMR (300 MHz, $\left.\mathrm{CDCl}_{3}\right) \delta$ : $7.27 \sim 7.08(\mathrm{~m}, 5 \mathrm{H}), 7.11(\mathrm{~d}, J=9.3 \mathrm{~Hz}, 1 \mathrm{H}), 6.03(\mathrm{~s}, 1 \mathrm{H})$, $5.98(\mathrm{~d}, J=9.3 \mathrm{~Hz}, 1 \mathrm{H}), 5.40$ (s, 1H), 4.99 (d, $J=11.7 \mathrm{~Hz}$, $1 \mathrm{H}), 4.90(\mathrm{~d}, J=11.7 \mathrm{~Hz}, 1 \mathrm{H}), 3.57$ (d, $J=10.5 \mathrm{~Hz}, 1 \mathrm{H})$, $3.14 \sim 2.96(\mathrm{~m}, 2 \mathrm{H}), 2.79(\mathrm{~d}, J=10.5 \mathrm{~Hz}, 1 \mathrm{H}), 2.30 \sim 2.05$ $(\mathrm{m}, 6 \mathrm{H}), 1.83 \sim 1.61(\mathrm{~m}, 2 \mathrm{H}), 1.51 \sim 1.38(\mathrm{~m}, 2 \mathrm{H}), 1.30 \sim$ $1.14(\mathrm{~m}, 3 \mathrm{H}), 1.26(\mathrm{~s}, 3 \mathrm{H}), 0.83(\mathrm{~d}, J=6.3 \mathrm{~Hz}, 3 \mathrm{H}) ;{ }^{13} \mathrm{C}$ NMR (75 MHz, $\left.\mathrm{CDCl}_{3}\right) \delta: 202.7,191.2,172.9,169.9$, $149.5,146.1,135.0,129.5,128.7,128.5,128.5,118.9$, $89.3,80.4,67.4,65.5,62.9,61.4,60.6,49.2,48.5,36.0$, $34.0,29.9,28.3,22.5,17.5,14.0,11.9$; TOF-MS $m / z(\%)$ : $519\left(\mathrm{M}^{+}+1,100\right), 497$ (25), 475 (18), 339 (20), 318 (29), 274 (80), 249 (65), 161 (24), 149 (42); HRMS calcd for $\mathrm{C}_{31} \mathrm{H}_{35} \mathrm{O}_{7}(\mathrm{M}+\mathrm{H})^{+}$519.2382, found 519.2347.

对映-3,15-二氧- $10 \beta$-羊基- $13 \beta$-异戊氧基-20-失碳赤 霉-1,16-二烯-7,19-二羧酸 7-苯甲酯 19,10-内酯(27): 产 率 78\%, m.p. $140 \sim 141{ }^{\circ} \mathrm{C} ;{ }^{1} \mathrm{H}$ NMR $\left(300 \mathrm{MHz}, \mathrm{CDCl}_{3}\right)$ $\delta: 7.25 \sim 7.05(\mathrm{~m}, 5 \mathrm{H}), 7.12(\mathrm{~d}, J=9.3 \mathrm{~Hz}, 1 \mathrm{H}), 6.03$ (s, 
1H), 5.98 (d, $J=9.3 \mathrm{~Hz}, 1 \mathrm{H}), 5.40$ (s, 1H), 4.99 (d, $J=$ $11.8 \mathrm{~Hz}, 1 \mathrm{H}), 4.90$ (d, $J=11.8 \mathrm{~Hz}, 1 \mathrm{H}), 3.56$ (d, $J=10.5$ $\mathrm{Hz}, 1 \mathrm{H}), 3.20 \sim 2.96(\mathrm{~m}, 2 \mathrm{H}), 2.79(\mathrm{~d}, J=10.5 \mathrm{~Hz}, 1 \mathrm{H})$, $2.30 \sim 2.05(\mathrm{~m}, 5 \mathrm{H}), 1.83 \sim 1.51(\mathrm{~m}, 3 \mathrm{H}), 1.41 \sim 1.28(\mathrm{~m}$, 2H), 1.26 (s, 3H), 0.81 (d, $J=6.5 \mathrm{~Hz}, 3 \mathrm{H}), 0.79$ (d, $J=6.5$ $\mathrm{Hz}, 3 \mathrm{H}) ;{ }^{13} \mathrm{C}$ NMR $\left(75 \mathrm{MHz}, \mathrm{CDCl}_{3}\right) \delta: 202.7,191.2$, $172.9,169.9,149.6,146.1,134.9,129.5,128.7,128.5$, $128.5,118.8,89.3,80.4,67.4,65.4,61.4,61.2,60.5,49.2$, $48.5,39.1,35.9,34.0,25.0,22.7,22.7,17.5,11.9$; TOF-MS $m / z(\%): 519\left(\mathrm{M}^{+}+1,100\right), 501$ (15), 411 (20), 339 (12), 149 (22); HRMS calcd for $\mathrm{C}_{31} \mathrm{H}_{35} \mathrm{O}_{7}(\mathrm{M}+\mathrm{H})^{+}$ 519.2382, found 519.2346.

\section{2 .4 抗肿瘤活性测试}

测试前用二甲亚砜(DMSO)分别溶解阳性对照化合 物 1、DDP 及样品 $8 \sim 27$, 并配成 $10 \mathrm{mg} / \mathrm{mL}$ 浓度的母液. 将对数生长期的细胞调整为适当浓度 (悬浮细胞 $6 \times 10^{4} / \mathrm{mL}$ ，贴壁细胞 $5 \times 10^{4} / \mathrm{mL}$ ) 后加入 96 孔培养板， $90 \mu \mathrm{L} /$ 孔. 药物设 5 个浓度, 每个浓度设 3 个平行孔, 10 $\mu \mathrm{L} /$ 孔，使受试浓度分别为 $0.01,0.1,1,10,100 \mu \mathrm{g} / \mathrm{mL}$; 空白孔为 $100 \mu \mathrm{L}$ 培养液, 阴性对照孔为 $90 \mu \mathrm{L}$ 的细胞加 $10 \mu \mathrm{L}$ 的培养液; 阳性对照药物为抗癌药物毛萼乙素、 顺铂. 贴壁生长细胞待培养 $24 \mathrm{~h}$ 贴壁后加药; 悬浮生长 细胞接种后即加药. 加药后置 $37{ }^{\circ} \mathrm{C}, 5 \% \mathrm{CO}_{2}$ 培养箱, 悬浮细胞培养 $48 \mathrm{~h}$, 贴壁细胞培养 $72 \mathrm{~h}$ 后, 加入 MTT (5 $\mathrm{mg} / \mathrm{mL}), 20 \mu \mathrm{L} /$ 孔, 继续培养 $4 \mathrm{~h}$ 后加入三联液 $[10 \mathrm{~g}$ $\mathrm{SDS}, 5 \mathrm{~mL}$ 异丁醇, $100 \mathrm{~mL} 0.012 \mathrm{~mol} / \mathrm{L}$ 的 $\mathrm{HCl}$ 溶液], 100 $\mu \mathrm{L} /$ 孔，放置过夜后，用酶标仪在 $570 \mathrm{~nm}$ 波长下测定各 孔的光密度 $(\mathrm{OD})$ 值. 用以下列公式计算细胞的生长抑 制率:

生长抑制率 $=(\mathrm{OD}$ 阴性对照 $-\mathrm{OD}$ 加药孔 $) / \mathrm{OD}$ 阴性对照 $\times 100 \%$.

LOGIT 法计算受试样品对细胞生长的半数抑制浓 度 $\mathrm{IC}_{50}$, 每个样品平行测定三次, 实验结果见表 2.

\section{References}

[1] (a) Mander, L. N. Chem. Rev. 1992, 92, 573.

(b) Mander, L. N. Nat. Prod. Rep. 2003, 20, 49.

[2] (a) MacMillan, J. Nat. Prod. Rep. 1997, 14, 221. (b) Hanson, J. R. Nat. Prod. Rep. 2006, 23, 875.

(c) Keyzers, R. A.; Peter, T. N.; Davies-Coleman, M. T. Nat. Prod. Rep. 2006, 23, 321.

(d) Hanson, J. R. Nat. Prod. Rep. 2007, 24, 1332.

[3] Sun, H.-D.; Huang, S.-X.; Han, Q.-B. Nat. Prod. Rep. 2006, 23, 673.

[4] (a) Zhao, Y.; Niu, X.-M; Qian, L.-P.; Liu, Z.-Y.; Zhao, Q.-S.; Sun, H.-D. Eur. J. Med. Chem. 2007, 42, 494.

(b) He, F.; Xiao, W.; Pu, J.; Wu, Y.; Zhang, H.; Li, X; Zhao, Y.; Yang, L.; Chen, G.; Sun, H. Phytochemistry 2009, 70, 1462.

(c) Aquila, S.; Weng, Z.-Y.; Zeng, Y.-Q.; Sun, H.-D.; Luis Rios, J. J. Nat. Prod. 2009, 72, 1269 .

(d) Mao, Z.; Li, Y.; Chen, J.; Wang, Y.; Zhang, H. Bioorg. Med. Chem. Lett. 2010, 20, 4116.

[5] (a) Ikezoe, T.; Yang, Y.; Bandobashi, K.; Saito, T.; Takemoto, S.; Machida, H.; Togitani, K.; Koeffler, H. P.; Taguchi, H. Mol. Cancer Ther. 2005, 4, 578 .

(b) Wang, L.; Zhao, W.-L.; Yan, J.-S.; Liu, P.; Sun, H.-P.; Zhou, G.-B.; Weng, Z.-Y.; Wu, W.-L.; Weng, X.-Q.; Sun, X.-J.; Chen, J.; Sun, H.-D.; Chen, S.-J. Cell Death Differ. 2007, 14, 306.

(c) Zhang, Y.-W.; Jiang, X.-X.; Chen, Q.-S.; Shi, W.-Y.; Wang, L.; Sun, H.-D.; Shen, Z.-X.; Chen, Z.; Chen, S.-J.; Zhao, W.-L. Exp. Hematol. 2010, 38, 191.

[6] Chen, J.; Sun, Z.; Zhang, Y.; Zeng, X.; Qing, C.; Liu, J.; Li, L.; Zhang, H. Bioorg. Med. Chem. Lett. 2009, 19, 5496.

[7] (a) MacMillan, J.; Wills, C. L. J. Chem. Soc., Perkin Trans. 11986 309.

(b) Lombardo, L.; Mander, L. N.; Turner, J. V. Aust. J. Chem. 1981, 34, 745 .

[8] Bhaskar, K. V.; Mander, L. N. Tetrahedron Lett. 1996, 37, 719.

[9] Dolan, S. C.; MacMillan, J. J. Chem. Soc., Perkin Trans. 1 1985, 2741.

[10] Liu, J.-P.; Zhang, H.-B.; Huang, S.-X.; Pu, J.-X.; Xiao, W.-L.; Zhang, X.-P.; Xiao, W.-D.; Lei, C.; Sun, H.-D. J. Heterocycl. Chem. 2012, 49, 571 .

[11] (a) Alley, M. C.; Scudiero, D. A.; Monks, A.; Hursey, M. L.; Czerwinski, M. J.; Fine, D. L.; Abbott, B. J.; Mayo, J. G.; Shoemaker, R. H.; Boyd, M. R. Cancer Res. 1988, 48, 589.

(b) Zhou, J.-J.; Yue, X.-F.; Han, J.-X.; Yang, W.-Y. Chin. J. Pharm. 1993, 24, 455. 